\title{
Delta C-13 Stratigraphy of the Proterozoic Bylot Supergroup, Baffin Island, Canada: Implications for Regional Lithostratigraphic Correlations
}

\section{Citation}

Kah, Linda C., Anne G. Sherman, Guy M. Narbonne, Andrew H. Knoll, and Alan J. Kaufman. 1999. Delta C-13 stratigraphy of the Proterozoic Bylot Supergroup, Baffin Island, Canada: Implications for regional lithostratigraphic correlations. Canadian Journal of Earth Sciences 36(3): 313-332.

\section{Published Version}

http://dx.doi.org/10.1139/cjes-36-3-313

\section{Permanent link}

http://nrs.harvard.edu/urn-3:HUL.InstRepos:3196275

\section{Terms of Use}

This article was downloaded from Harvard University's DASH repository, and is made available under the terms and conditions applicable to Other Posted Material, as set forth at http:// nrs.harvard.edu/urn-3:HUL.InstRepos:dash.current.terms-of-use\#LAA

\section{Share Your Story}

The Harvard community has made this article openly available.

Please share how this access benefits you. Submit a story.

\section{Accessibility}




\title{
$\delta^{13} \mathrm{C}$ stratigraphy of the Proterozoic Bylot Supergroup, Baffin Island, Canada: implications for regional lithostratigraphic correlations
}

\author{
Linda C. Kah, Anne G. Sherman, Guy M. Narbonne, Andrew H. Knoll, and \\ Alan J. Kaufman
}

\begin{abstract}
The Bylot Supergroup, northern Baffin Island, contains $>1500 \mathrm{~m}$ of platform, shelf, and slope carbonates deposited between $\sim 1270$ and $\sim 723$ Ma. Limited chronostratigraphic data have led to the broad correlation of the Bylot Supergroup with predominantly Neoproterozoic successions in northern and western Laurentia; yet, detailed correlation has been impossible given biostratigraphic and lithostratigraphic limitations. Carbon-isotope chemostratigraphy represents a potential dataset to constrain such interregional correlations. Carbon isotopic data from the Bylot Supergroup and broadly coeval successions from Somerset Island and northwest Greenland reveal distinct stratigraphic trends in $\delta^{13} \mathrm{C}$, with intervals of moderate ${ }^{13} \mathrm{C}$ enrichment $(+3.5 \pm 1 \%$ o) punctuated by excursions to slightly negative values $(-1.0 \pm 1 \%$ o). Although the scale of the observed variation is muted relative to Neoproterozoic standards, the dissimilarity of values to those recorded in northwestern Laurentia suggests that these strata delineate a discrete depositional interval. Comparison of isotopic values with published data indicates that $\delta^{13} \mathrm{C}$ values between approximately -1.0 and $+4.0 \%$ are characteristic of the interval between $\sim 1300$ and $\sim 800 \mathrm{Ma}$. This pattern is distinct from that of younger Neoproterozoic successions, which typically record values $>+5 \%$, and older Mesoproterozoic successions, which record values near $0 \%$, and suggests that these moderately positive values may be useful for broad time correlation. Compilation of new and published data permits the tentative reconstruction of a global Mesoproterozoic carbon isotopic curve.
\end{abstract}

Résumé : Le Supergroupe de Bylot, dans le nord de l'île de Baffin, est formé de $>1500 \mathrm{~m}$ de carbonates qui furent déposés sur une plate-forme continentale et un talus, entre $\sim 1270$ et $\sim 723$ Ma. Les données chronostratigraphiques limitées n'autorisent qu'une corrélation d'ordre général entre le Supergroupe de Bylot et les successions principalement néoprotérozoïques des régions septentrionales et occidentales de Laurentia; jusqu'à présent, il a été impossible d'établir une corrélation détaillée en raison du petit nombre de données biostratigraphiques et lithostratigraphiques. La chimiostratigraphie des isotopes du $\mathrm{C}$ a fourni un ensemble de données pouvant aider à mieux définir les corrélations inter-régionales. Les résultats des analyses d'isotopes du C pour le Supergroupe de Bylot et les successions grossièrement contemporaines de l'île Somerset et du nord-ouest du Groenland révèlent des tendances stratigraphiques distinctes dans les valeurs de $\delta^{13} \mathrm{C}$, avec des intervalles d'enrichissement modéré de ${ }^{13} \mathrm{C}(+3,5 \pm 1 \%$ ) , ponctués d'incursions de valeurs légèrement négatives $(-1,0 \pm 1 \%$ ). Même si l'échelle de la variation observée est atténuée par rapport aux standards du Néoprotérozoïque, les valeurs différentes de celles enregistrées dans le nord-ouest de Laurentia suggèrent que ces strates représentent un intervalle de sédimentation discontinue. La comparaison des données isotopiques avec celles déjà publiées indique que les valeurs de $\delta^{13} \mathrm{C}$ comprises entre environ $-1,0$ et $+4,0 \%$ o caractérisent la période échelonnée entre $\sim 1300$ et $\sim 800$ Ma. Cette particularité est absente dans les successions néoprotérozoïques les plus jeunes qui fournissent spécifiquement des valeurs $>+5 \%$, et des successions néoprotérozoïques les plus vieilles dont les valeurs oscillent autour de $0 \%$, et elle suggère en plus que ces valeurs modérément positives peuvent être utiles pour établir grosso modo une corrélation-temps. La compilation de ces données nouvelles jointe à celles publiées autorise une révision de la courbe globale des isotopes du $\mathrm{C}$ au Mésoprotérozoïque.

[Traduit par la Rédaction]

Received April 1, 1998. Accepted November 4, 1998.

L.C. Kah ${ }^{1}$ and A.H. Knoll. Department of Earth and Planetary Sciences, Harvard University, Cambridge, MA 02138, U.S.A. A.G. Sherman and G.M. Narbonne. Department of Geological Sciences, Queen's University, Kingston, ON K1A 07L, Canada. A.J. Kaufman. Department of Geology, The University of Maryland, College Park, MD 20742, U.S.A.

${ }^{1}$ Corresponding author. Present address: Department of Geological Sciences, University of Tennessee, Knoxville, TN 37996, U.S.A. (e-mail: lckah@alum.mit.edu). 
Fig. 1. Regional setting and stratigraphy of the Bylot Supergroup (BY), modified from Jackson and Iannelli (1981). Eqalulik and Uluksan group sediments of the Bylot Supergroup may be genetically related to lithologically similar sediments across the eastern Arctic platform, including the Aston and Hunting formations (AH), Fury and Hecla Group (FH), and Thule Group (TG) of northwest Greenland. Asterisks mark horizons with paleomagnetic poles determined by Fahrig et al. (1981): 1, Mackenzie pole (1270 \pm 2 Ma; LeCheminant and Heaman 1991); 2, probable Sudbury pole (1238 \pm 4 Ma; Krogh et al. 1987); 3, possible Logan Sill pole $\left(1109_{-2}^{+4}\right.$ (Ma; Krogh et al. 1987); 4, Franklin pole (723 \pm 3 Ma; Heaman et al. 1990).

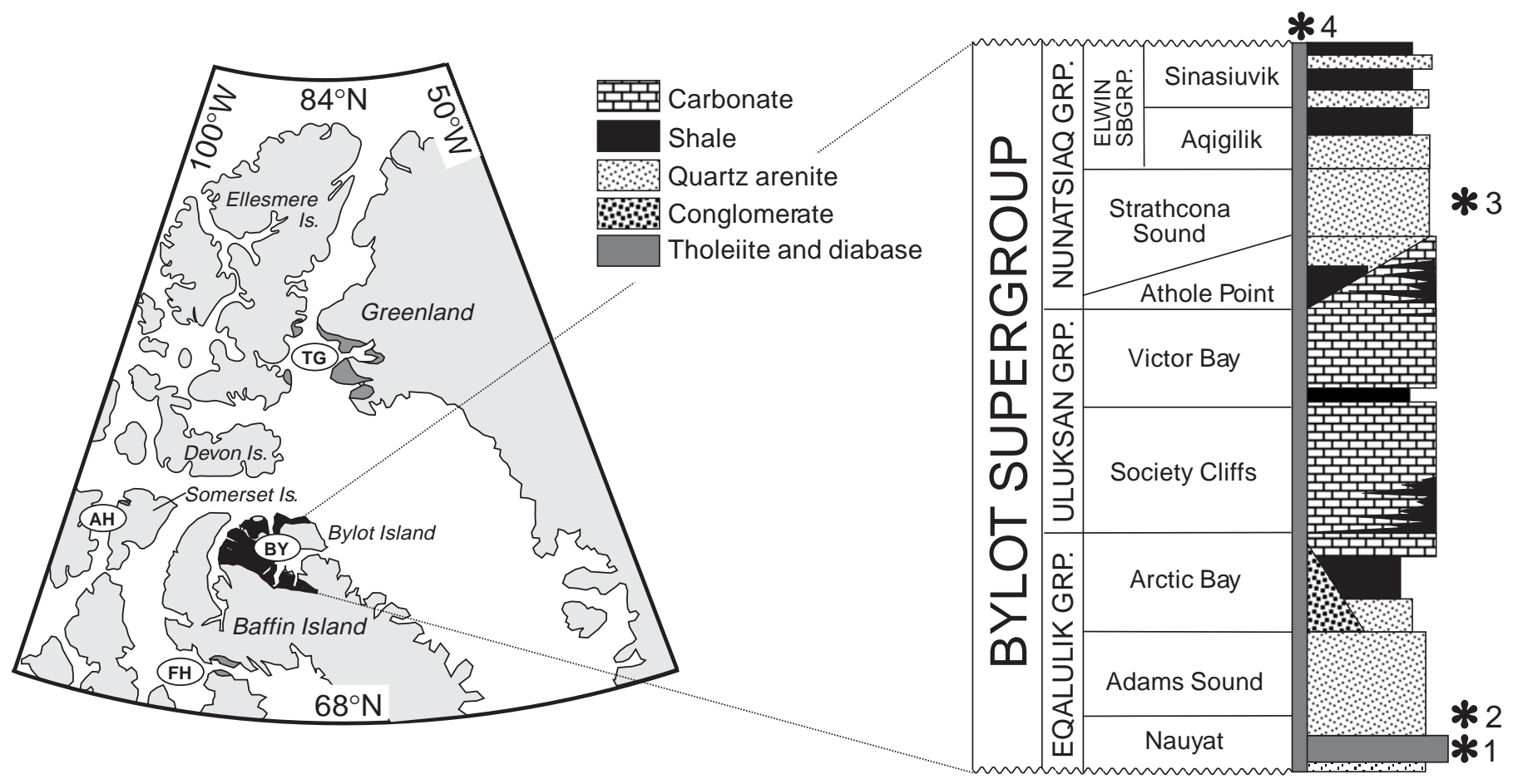

\section{Introduction}

Studies of Neoproterozoic (1000-543 Ma) successions worldwide have demonstrated that fine-grained marine carbonates, including both limestone and dolostone, can faithfully record the carbon isotopic composition of coeval seawater (Kaufman and Knoll 1995, and references therein). Secular variation in this record, generated by temporal changes in the relative rates of input and export of organic and inorganic carbon to the ocean-atmosphere system, provides a means of stratigraphic correlation independent of standard biostratigraphic techniques. Such techniques have proven especially useful in Proterozoic successions for which radiometric and biostratigraphic data are limited or inconclusive. Comparison of data with a global Neoproterozoic carbon isotopic curve has proven successful in confidently correlating interbasinal successions (Knoll and Walter 1992; Narbonne et al. 1994), in evaluating intrabasinal depositional and diagenetic trends (Pelechaty et al. 1996), and in determining the relative timing of environmental (Kaufman et al. 1997; Hoffman et al. 1998), and evolutionary events (Narbonne et al. 1994; Brasier et al. 1996).

Our understanding of older successions, however, is significantly limited. The Mesoproterozoic Eon (1600$1000 \mathrm{Ma}$ ) represents a critical period in Earth history from the standpoint of eukaryotic diversification (Knoll 1991), fundamental transitions in the oxidation state of the Earth's atmosphere and oceans (Des Marais et al. 1992), and global tectonic reorganization (Hoffman 1991; Dalziel 1991), yet relatively few studies have produced data that are stratigraphically and diagenetically well constrained. The paucity of data, as well as limited range of isotopic variation observed in older Mesoproterozoic successions (Buick et al. 1995; Xiao et al. 1997; Frank et al. 1997; Brasier and Lindsay 1998), severely limits our ability to evaluate even first-order secular variation.

In this paper we provide carbon isotopic data for several late Mesoproterozoic to earliest Neoproterozoic successions in eastern Arctic Canada. We utilize an integrated approach in which samples are evaluated for possible diagenetic effects and interpreted within the framework of both stratigraphy and depositional environment. This approach not only permits recognition of primary isotopic values but also allows discrimination between local and global isotopic variation; resulting data are then used to evaluate regional correlations. By combining new and published data, we construct a preliminary global isotopic reference curve extending through both the Mesoproterozoic and Neoproterozoic eras. Refinement of this global curve by the addition of new data and radiometric constraints will provide the framework for understanding the complex biogeochemical interactions active during this critical period of Earth's history.

\section{Geologic setting}

The Bylot Supergroup is a thick succession $(\sim 6000 \mathrm{~m})$ of nearly undeformed, late Mesoproterozoic to earliest Neoproterozoic sediments exposed within the fault-bounded Borden Basin of northernmost Baffin and Bylot islands (Fig. 1). The supergroup is composed of three lithostrati- 
Fig. 2. Location map for Bylot Supergroup carbonates. Samples for geochemical analysis were collected from measured sections at Mala River (MR), Tremblay Sound (TS), Milne Inlet (MI), and White Bay (WB).

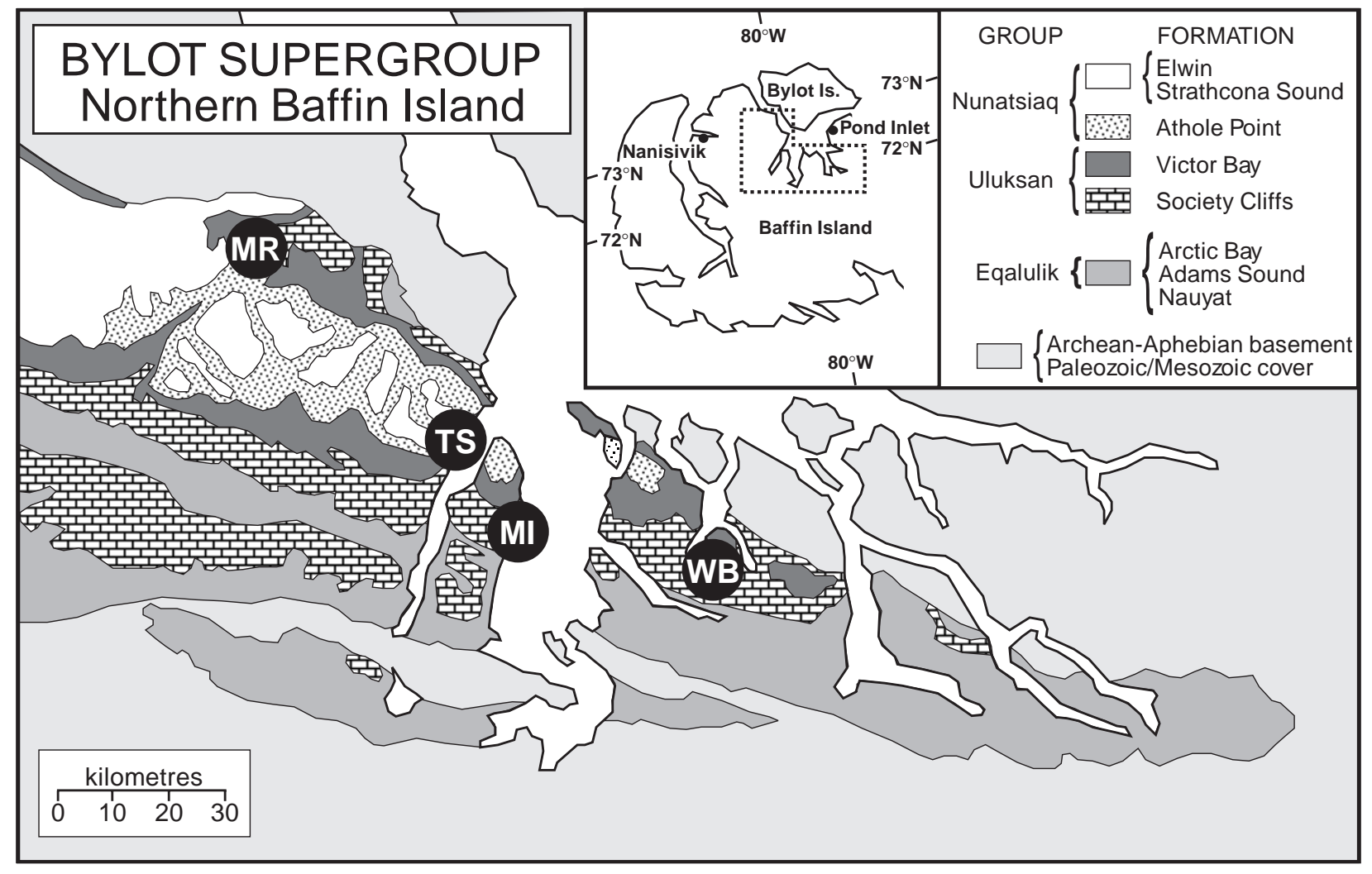

graphic packages, separated by regional unconformities, that reflect the combined tectonic and subsidence history of the basin. The Society Cliffs, Victor Bay, and Athole Point formations consist of a variety of platform, shelf, and slope carbonates. Carbonate rocks unconformably overlie fluvial to marine volcanic and siliciclastic rocks of the Eqalulik Group and interfinger with (Athole Point) or are unconformably overlain by (Victor Bay) subtidal marine sandstone, silt, and shale of the Nunatsiaq Group (Jackson and Iannelli 1981). For detailed descriptions of Bylot Supergroup siliciclastic rocks, refer to Iannelli (1979), Jackson and Iannelli (1981), Jackson et al. (1975), and Knight and Jackson (1994).

On Baffin Island, the Society Cliffs, Victor Bay, and Athole Point formations are exposed in linear, east-westtrending belts (Fig. 2); strata show only shallow dips, are structurally undeformed, and are affected only by regional subgreenschist-grade metamorphism. These formations comprise a $>1500 \mathrm{~m}$ thick section of carbonates and subordinate siliciclastics deposited in a range of subtidal to supratidal environments. More than $1200 \mathrm{~m}$ of peritidal evaporite, carbonate, and siliciclastic strata also occurs on northern and western Bylot Island (Jackson and Davidson 1978), where lithologic similarities have prohibited formation-scale subdivision (Jackson et al. 1985).

The Society Cliffs Formation consists of shallow subtidal to supratidal carbonate and minor siliciclastic rocks that gradationally to unconformably overlie interbedded dolostone and black shale of the Arctic Bay Formation (Jackson and Iannelli 1981). Intertidal to supratidal dolostone lithologies are dominant in the eastern portions of Borden Basin, where 10-50 m thick shoaling cycles com- prised of organic-rich stromatolitic and microbially laminated dolostone and laminated sea-floor precipitates interfinger with bedded evaporites and terrigenous shoreline deposits to the east and north. Microbenthic fossils preserved in early diagenetic chert (Hofmann and Jackson 1991), desiccation features, gypsum, and rare halite indicate deposition under marine to hypersaline conditions. In the central basin, Society Cliffs carbonates consist of a heterogeneous package of intraclastic-oolitic grainstone, columnar, domal, and conical stromatolites, and thinly bedded dolostone. Grainstones are most abundant in the Milne Inlet region where they comprise 1-10 m shoaling cycles capped by tepee cracks, indicating episodic exposure. To the west, cycles become more thinly bedded and organic rich, reflecting shallow subtidal depositional conditions (Olson 1984).

The Victor Bay Formation comprises a variety of carbonate and siliciclastic lithologies deposited in a range of peritidal shelf to subtidal shelf and slope environments (Jackson and Iannelli 1981; Narbonne and James 1996). The transition from peritidal carbonates of the Society Cliffs is marked by an abrupt transition to black to dark grey calcareous shales (Jackson and Iannelli 1981). This transition is conformable through much of Borden Basin, except in the west, where an episode of karst formation may have occurred prior to deposition of the Victor Bay Formation (Geldsetzer 1973; Clayton and Thorpe 1982; Olson 1984). Black shale is thickest in the central region of Borden Basin, thins dramatically to the east, and is absent to the north and on Bylot Island (Jackson and Iannelli 1981). Thin calcareous and dolomitic interbeds increase in thickness and number upwards as Victor Bay strata pass upwards into dominantly 
subtidal, carbonate lithologies. In the central basin, Victor Bay lithologies include pyritic shale, stromatolitic carbonate, and ribbon and nodular limestones that contain abundant slump features, suggesting deposition in subtidal outer-ramp environments. Northern facies are composed of microbially laminated dolostone, calcareous mudstone with abundant molar tooth structure (James et al. 1998), and subordinate nodular and ribbon limestone. Thin stromatolitic beds and mud-cracked red shales, though uncommon, attest to deposition in shallower water than that of correlative sections in the central basin.

The Athole Point Formation is present only in the eastern regions of Borden Basin; it interfingers with and is replaced by siliciclastic facies of the Strathcona Sound Formation to the north and west (Jackson and Ianelli 1981) and is separated from underlying formations by a regional unconformity (Jackson et al. 1978; Jackson and Ianelli 1981; Narbonne and James 1996). In central Borden Basin, the lower Athole Point consists of recessively weathered calcareous shale and shaley limestone and subordinate quartz-rich, calcareous turbidites. Calcareous turbidites predominate in the upper Athole Point, and beds of reddish sandstone become increasingly abundant as the contact with the overlying Strathcona Sound is approached. To the north, the lower Athole Point resembles the underlying Victor Bay, containing various shallow subtidal stromatolitic, oolitic, and thinly laminated microbial facies. Stratigraphically higher facies, however, are lithologically identical to the upper Athole Point in the central basin, consisting of thinly bedded calcareous shales overlain by a thick package of calcareous turbidites and quartz sandstone.

\section{Age}

The age of the Bylot Supergroup remains poorly constrained. Paleomagnetic and geochemical evidence suggests that basal Nauyat volcanics are genetically related to the widespread Mackenzie igneous event (Fahrig et al. 1981; Galley et al. 1983). Nauyat basalts yielded a paleomagnetic pole identical to that of Mackenzie intrusions (Fahrig et al. 1981) which elsewhere give an age of $1267 \pm 2 \mathrm{Ma}$ (U-Pb baddeleyite; LeCheminant and Heaman 1989). This date, broadly supported by $\mathrm{Rb}-\mathrm{Sr}$ (1129 Ma, no error reported; Knight and Jackson 1994) and K-Ar ages (ranging from $762 \pm 26$ to $1221 \pm 31$ Ma; Jackson 1974; Jackson and Iannelli 1981) on Nauyat Formation basalts, provides an approximate age for initiation of Bylot sedimentation. Somewhat younger radiometric ages obtained from the Nauyat basalts may be related to regional greenschist stage metamorphism (Jackson et al. 1990). Diabase intrusions, which cut the entire sedimentary package, contain measured paleomagnetic poles which indicate that they are part of the widespread Franklin intrusive event (Fahrig et al. 1971, 1981; Christie and Fahrig 1983; Pehrsson and Buchan 1993), elsewhere dated at $723 \pm 3 \mathrm{Ma}$ (U-Pb baddeleyite; Heaman et al. 1990).

The age of the Bylot Supergroup therefore lies between $1267 \pm 2$ and $723 \pm 3 \mathrm{Ma}$, placing the succession within Sequence B of Young (1979). The similarity of Strathcona Sound paleomagnetic poles both to those of the Nauyat Formation and to the well-constrained Logan Sill pole
(1109 +4 Ma, U-Pb baddeleyite; Krogh et al. 1987), however, suggests that the Bylot Supergroup may have been deposited wholly within the Mesoproterozoic (Fahrig et al. 1981; Knight and Jackson 1994). If these estimates are correct, it is unlikely that Bylot Supergroup deposition was coeval with that of the Shaler Supergroup and correlatives of Sequence $\mathrm{B}$, which have maximum ages between $1077 \pm 3$ and $1003 \pm 3$ Ma (U-Pb detrital zircon; Rainbird et al. 1996). Biostratigraphic evidence cannot further constrain depositional ages; acritarch taxa from the Bylot Supergroup consist largely of unornamented leiosphaerids (Hofmann and Jackson 1994). These taxa, common in Mesoproterozoic successions, are typically long-ranged and thus make poor stratigraphic markers.

\section{Geochemical analyses}

Elemental and isotopic analyses from 84 samples were used to establish a chemostratigraphic curve. Samples were collected at 10-20 m intervals through dominantly subtidal lithologies exposed along Milne Inlet and Tremblay Sound (Fig. 2). A smaller group of 33 samples was collected from laterally equivalent successions at White Bay and Mala River. Society Cliffs and Victor Bay strata in these regions were deposited largely in peritidal environments and were collected to assess intrabasinal isotopic variation. Additionally, 20 analyses from the Hunting Formation, Somerset Island, and 12 previously unpublished analyses from the Narssârssuk Formation, Thule Group, Greenland, are provided for comparison.

Diagenetic alteration is of particular concern when constructing an isotopic profile of seawater variation. We therefore conducted several tests designed to assure sampling of least altered sedimentary components. At the earliest stage, standard petrographic and cathodoluminescence techniques were utilized to assess the diagenetic history of samples prior to geochemical analysis (cf. Kaufman et al. 1991; Kaufman and Knoll 1995). Petrographic analysis permits characterization of primary and secondary sedimentary components within samples. Petrographic features such as stylolites, void-filling sparry cements associated with veins and dissolutional features, and diagenetic clay mineralization reflect postdepositional alteration and were avoided during sampling. Additionally, occurrences and abundances of siliciclastic material within the carbonate matrix were noted. Siliciclastic material commonly is associated with increased permeability to diagenetic fluids and can therefore presage diagenetic alteration. Furthermore, siliciclastic material can react with carbonate under a broad range of thermal conditions, resulting in overprinting of primary isotopic signatures.

Carbonate components were also evaluated for degree of recrystallization. Diagenetic and neomorphic recrystallization processes frequently result in an increase in the average grain size of carbonates or a complete obliteration of primary fabrics. Empirical evidence suggests that primary isotopic compositions may be altered during this process as well (Fairchild et al. 1990; Fairchild and Spiro 1987). Finegrained, primary depositional components, such as micrites, syndepositional marine cements, oolitic grains, and finegrained carbonate material within stromatolites are regarded 
as least likely to have undergone diagenetic recrystallization and (or) neomorphism, and therefore most likely to retain primary isotopic signatures. All calcareous samples from the Victor Bay and Athole Point formations are extremely fine grained, composed predominantly of micritic and microsparitic carbonate. Dolomitic microbial, oolitic, and precipitated carbonate from the Society Cliffs Formation is also dominantly fine grained and preserves distinct morphologies of originally aragonitic fabric elements, indicating dolomitic replacement prior to early diagenetic aragonite to calcite transformations.

Cathodoluminescence (CL) petrography of polished thick sections provided an additional estimate of alteration that may not have been apparent during routine petrographic analysis. Luminescence in sedimentary carbonates commonly is activated by the presence of $\left[\mathrm{Mn}^{2+}\right]$ within the carbonate lattice and quenched by $\left[\mathrm{Fe}^{2+}\right]$ (Hemming et al. 1989). Because $\mathrm{Mn}$ and $\mathrm{Fe}$ are common additives to sedimentary carbonates under both meteoric and burial diagenetic conditions, cathodoluminescence provides a qualitative estimate of diagenetic alteration. The reliability of CL in determining diagenetic histories, however, must always be regarded with caution. Dull luminescence can result from even low abundances of iron (Marshall 1992; Savard et al. 1995) and may not accurately foretell diagenetic alteration (cf. Rush and Chafetz 1990). Despite uncertainties, CL provides a firstorder evaluation technique; whereas texturally preserved material typically records uniform or clearly zoned luminescence, small-scale heterogeneities commonly result from recrystallization.

Calcareous and dolomitic samples from the Victor Bay and Athole Point formations revealed only homogenous, dull luminescence, consistent with their homogenous grain size. Compositionally heterogeneous peritidal dolostones of the Society Cliffs Formation displayed a greater range of luminescence; depositional components including ooids, micrite, micritic intraclasts, and syndepositional sea-floor cements revealed dull to moderate luminescence, whereas void-filling cements within fenestrae were characterized by high luminescence. Although greater overall luminosity was recorded in peritidal facies, distinct CL boundaries between depositional components and void-filling cements, and the absence of small-scale heterogeneous textures, suggest minimal diagenetic overprinting of primary components.

Following petrographic and cathodoluminescence analysis, least-altered components within each sample were selected for microsampling. Components included ooids (MI), micrite and micritic intraclasts (MI, WB, TS, MR), microsparitic microbial laminations (MI, WB, TS, MR), and syndepositional sea-floor precipitates (WB). Where appropriate, multiple phases within a single thick section were sampled to evaluate isotopic variation among primary depositional components. Dual-phase sampling included ooids and surrounding micrite (MI-3a, MR-3b, MI-40a, MI40b), sea-floor precipitates and micritic drapes (WB-12a, WB-12b), and microbial laminations and molar-tooth cements (MR-3a, MR-3b, MR-9a, MR-9b).

Selected sites were drilled with a $1 \mathrm{~mm}$, diamondtipped drill bit, and resulting powder was collected for elemental and isotopic analysis. Analytical procedures follow those described in detail by Kaufman et al. (1991),
Fig. 3. Summary of analytical procedures, modified from Kaufman and Knoll (1995).

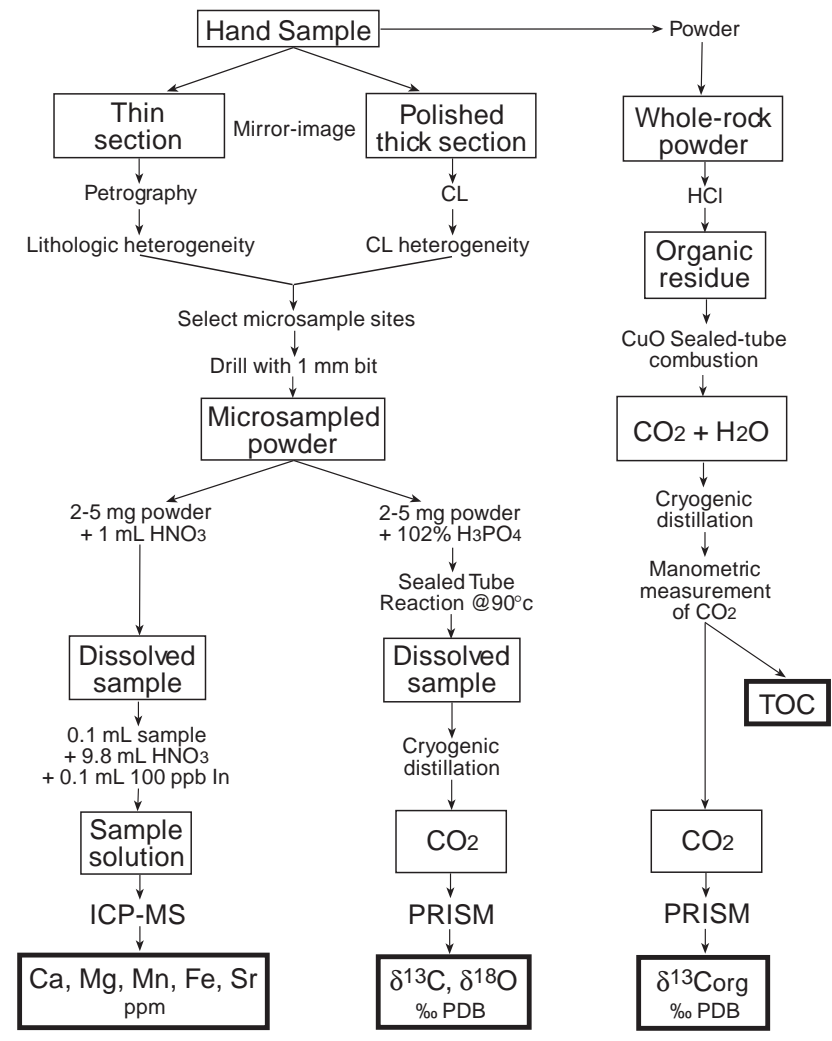

Derry et al. (1992), Narbonne et al. (1994), and Kaufman and Knoll (1995), and are summarized in Fig. 3. All values are reported in $\delta$ notation relative to a Peedee belemnite (PDB) standard, with precisions to $0.1 \%$ o for carbon and $0.3 \%$ for oxygen based on multiple analyses of an internal laboratory standard. Results of elemental and isotopic analyses of Bylot Supergroup carbonate rocks are provided in Tables 1 and 2.

\section{Discussion}

\section{Constraints on postdepositional diagenesis}

Bylot Supergroup samples typically show a range of isotopic values (Fig. 4). Although $\delta^{13} \mathrm{C}$ versus $\delta^{18} \mathrm{O}$ cross-plots reveal considerable isotopic variability, nearly nonoverlapping $\delta^{13} \mathrm{C}$ values between the Society Cliffs Formation and combined Victor Bay and Athole Point datasets suggest that $\delta^{13} \mathrm{C}$ values may provide effective stratigraphic markers. Yet before secular variation can be established, alternate possibilities for isotopic variability must be explored.

During postdepositional diagenesis, carbonate rocks typically show more negative $\delta^{13} \mathrm{C}$ and $\delta^{18} \mathrm{O}$ values accompanied by increased $\mathrm{Mn}$ and $\mathrm{Fe}$ concentrations and decreased $\mathrm{Sr}$ concentrations. In the absence of $\mathrm{HCO}_{3}{ }^{-}$-rich fluid migration, carbon isotopic values are typically buffered by initial dissolution and recrystallization of the carbonate phase, resulting in little alteration of primary $\delta^{13} \mathrm{C}$ compositions (Kaufman et al. 1991). Even postdepositional thermal alteration of organic matter often preserves primary carbon isotopic signatures in carbonate phases (Wickham and Peters 1993). In contrast, oxygen isotopic depletion, which results 
Table 1. Isotopic and elemental compositions, Milne Inlet and Tremblay Sound.

\begin{tabular}{|c|c|c|c|c|c|c|c|c|}
\hline $\begin{array}{l}\text { Sample } \\
\text { No. }^{a}\end{array}$ & Formation & $\begin{array}{l}\text { Height } \\
\text { (m) }\end{array}$ & $\begin{array}{l}\delta^{13} \mathrm{C} \\
(\% \circ \mathrm{PDB})\end{array}$ & $\begin{array}{l}\delta^{18} \mathrm{O} \\
(\% \circ \mathrm{PDB})^{b}\end{array}$ & $\mathrm{Mg} / \mathrm{Ca}$ & $\begin{array}{l}\mathrm{Fe} \\
(\mathrm{ppm})\end{array}$ & $\begin{array}{l}\mathrm{Mn} \\
(\mathrm{ppm})\end{array}$ & $\begin{array}{l}\mathrm{Sr} \\
(\mathrm{ppm})\end{array}$ \\
\hline MI-01 & Society Cliffs & 68 & 3.4 & -5.0 & 0.29 & 1022 & 147 & 79 \\
\hline MI-02 & Society Cliffs & 72 & 3.8 & -4.0 & 0.34 & 1034 & 124 & 77 \\
\hline MI-03a & Society Cliffs & 83 & 3.9 & -3.9 & 0.34 & 911 & 77 & 69 \\
\hline MI-03b & Society Cliffs & 83 & 4.0 & -2.7 & 0.34 & 805 & 127 & 72 \\
\hline MI-04 & Society Cliffs & 93 & 4.0 & -4.4 & 0.35 & 783 & 95 & 51 \\
\hline MI-05 & Society Cliffs & 106 & 4.0 & -4.9 & 0.35 & 872 & 76 & 56 \\
\hline MI-06 & Society Cliffs & 114 & 4.1 & -2.2 & 0.52 & 1394 & 30 & 38 \\
\hline MI-07 & Society Cliffs & 121 & 4.0 & -3.0 & 0.50 & 1082 & 63 & 36 \\
\hline MI-08 & Society Cliffs & 131 & 3.9 & -7.3 & 0.56 & 1203 & 51 & 25 \\
\hline MI-09 & Society Cliffs & 140 & 3.5 & -5.8 & 0.56 & 1057 & 107 & 32 \\
\hline MI-10 & Society Cliffs & 150 & 3.5 & -4.2 & 0.55 & 1070 & 49 & 43 \\
\hline MI-11 & Society Cliffs & 171 & 3.8 & -3.1 & 0.57 & 1135 & 38 & 45 \\
\hline MI-12 & Society Cliffs & 181 & 3.6 & -6.2 & 0.53 & 1075 & 57 & 143 \\
\hline MI-14 & Society Cliffs & 202 & 3.7 & -8.0 & 0.75 & 200 & 24 & 14 \\
\hline MI-15 & Society Cliffs & 216 & 3.6 & -3.9 & 0.33 & 1031 & 106 & 76 \\
\hline MI-16 & Society Cliffs & 224 & 3.4 & -3.7 & 0.37 & 1875 & 78 & 90 \\
\hline MI-17 & Society Cliffs & 236 & 3.2 & -9.0 & 0.33 & 1464 & 104 & 71 \\
\hline MI-18 & Society Cliffs & 244 & 3.3 & -12.4 & 0.40 & 2487 & 96 & 83 \\
\hline MI-19 & Society Cliffs & 253 & 3.5 & -9.8 & 0.45 & 460 & 64 & 24 \\
\hline MI-20 & Society Cliffs & 260 & 3.6 & -6.9 & 0.47 & 302 & 36 & 28 \\
\hline MI-21 & Society Cliffs & 271 & 3.6 & -5.6 & 0.50 & 395 & 30 & 44 \\
\hline MI-22 & Society Cliffs & 284 & 3.2 & -5.8 & 0.56 & 71 & 39 & 24 \\
\hline MI-23 & Society Cliffs & 291 & 3.5 & -3.8 & 0.49 & 390 & 43 & 41 \\
\hline MI-24 & Society Cliffs & 300 & 3.6 & -6.2 & 0.51 & 359 & 67 & 37 \\
\hline MI-25 & Society Cliffs & 310 & 3.5 & -6.8 & 0.52 & 385 & 64 & 30 \\
\hline MI-26 & Society Cliffs & 322 & 3.3 & -3.4 & 0.49 & 488 & 71 & 41 \\
\hline MI-27 & Society Cliffs & 331 & 3.5 & -7.6 & 0.50 & 480 & 62 & 27 \\
\hline MI-28 & Society Cliffs & 340 & 3.4 & -5.6 & 0.49 & 471 & 70 & 30 \\
\hline MI-29 & Society Cliffs & 356 & 3.6 & -3.2 & 0.50 & 465 & 81 & 41 \\
\hline MI-30 & Society Cliffs & 362 & 3.4 & -5.1 & 0.48 & 479 & 49 & 37 \\
\hline MI-31 & Society Cliffs & 372 & 3.5 & -3.6 & 0.48 & 489 & 40 & 42 \\
\hline MI-32 & Society Cliffs & 382 & 3.4 & -8.4 & 0.36 & 400 & 76 & 29 \\
\hline MI-33 & Society Cliffs & 392 & 3.1 & -5.7 & 0.48 & 492 & 79 & 31 \\
\hline MI-35 & Society Cliffs & 410 & 3.4 & -3.8 & 0.51 & 333 & 78 & 36 \\
\hline MI-36 & Society Cliffs & 434 & 3.2 & -2.7 & 0.48 & 512 & 76 & 39 \\
\hline MI-37 & Society Cliffs & 443 & 3.2 & -6.2 & 0.49 & 524 & 130 & 37 \\
\hline MI-38 & Society Cliffs & 451 & 3.7 & -2.4 & 0.45 & 500 & 37 & 69 \\
\hline MI-39 & Society Cliffs & 463 & 3.2 & -3.8 & 0.46 & 451 & 78 & 46 \\
\hline MI-40a & Society Cliffs & 471 & 3.2 & -2.1 & 0.46 & 526 & 60 & 53 \\
\hline MI-40b & Society Cliffs & 471 & 3.1 & -6.1 & 0.47 & 421 & 80 & 46 \\
\hline MI-41 & Society Cliffs & 483 & 2.9 & -2.8 & 0.42 & 446 & 43 & 46 \\
\hline TS-1 & Victor Bay & 580 & 0.3 & -6.8 & 0.02 & 1000 & 21 & 524 \\
\hline TS-2 & Victor Bay & 597 & 1.6 & -5.7 & 0.06 & 1153 & 42 & 548 \\
\hline TS-3 & Victor Bay & 603 & -0.4 & -6.1 & 0.09 & 2077 & 112 & 932 \\
\hline TS-4 & Victor Bay & 603 & -0.1 & -4.2 & 0.19 & 3805 & 145 & 298 \\
\hline TS-5 & Victor Bay & 612 & 0.3 & -7.0 & 0.38 & 2914 & 208 & 171 \\
\hline TS-6 & Victor Bay & 627 & 0.9 & -4.9 & 0.01 & 854 & 12 & 1724 \\
\hline TS-7 & Victor Bay & - & - & - & 0.08 & 2974 & 164 & 677 \\
\hline TS-8 & Victor Bay & 639 & 0.2 & -6.3 & 0.13 & 2453 & 240 & 562 \\
\hline TS-9 & Victor Bay & 674 & 0.8 & -1.9 & 0.44 & 844 & 196 & 107 \\
\hline TS-10 & Victor Bay & 691 & 0.1 & -6.1 & 0.08 & 1597 & 83 & 573 \\
\hline TS-11 & Victor Bay & 718 & 0.7 & -4.9 & 0.05 & 1925 & 26 & 333 \\
\hline TS-13 & Victor Bay & 748 & 0.3 & -7.5 & 0.17 & 1444 & 79 & 408 \\
\hline TS-14 & Victor Bay & 748 & 1.1 & -6.3 & 0.39 & 981 & 141 & 70 \\
\hline TS-15 & Victor Bay & 770 & 1.3 & -5.3 & 0.56 & 1227 & 60 & 54 \\
\hline TS-16 & Victor Bay & 804 & 1.3 & -0.9 & 0.62 & 418 & 18 & 26 \\
\hline
\end{tabular}


Table 1 (concluded).

\begin{tabular}{|c|c|c|c|c|c|c|c|c|}
\hline $\begin{array}{l}\text { Sample } \\
\text { No. }{ }^{a}\end{array}$ & Formation & $\begin{array}{l}\text { Height } \\
\text { (m) }\end{array}$ & $\begin{array}{l}\delta^{13} \mathrm{C} \\
(\% \circ \mathrm{PDB})\end{array}$ & $\begin{array}{l}\delta^{18} \mathrm{O} \\
(\% \circ \mathrm{PDB})^{b}\end{array}$ & $\mathrm{Mg} / \mathrm{Ca}$ & $\begin{array}{l}\mathrm{Fe} \\
(\mathrm{ppm})\end{array}$ & $\begin{array}{l}\mathrm{Mn} \\
(\mathrm{ppm})\end{array}$ & $\begin{array}{l}\mathrm{Sr} \\
(\mathrm{ppm})\end{array}$ \\
\hline TS-17 & Victor Bay & 825 & 1.3 & -4.4 & 0.56 & 1327 & 124 & 70 \\
\hline TS-18 & Victor Bay & 838 & 1.2 & -1.6 & 0.55 & 1185 & 44 & 75 \\
\hline TS-19 & Victor Bay & 846 & 1.0 & -9.8 & 0.56 & 1309 & 78 & 42 \\
\hline TS-21 & Victor Bay & 881 & 1.7 & -3.9 & 0.56 & 1164 & 42 & 72 \\
\hline TS-22 & Athole Point & 920 & 2.1 & -1.1 & 0.56 & 1122 & 87 & 92 \\
\hline $\mathrm{TS}-25$ & Athole Point & 934 & 1.6 & -6.8 & 0.02 & 1564 & 25 & 321 \\
\hline TS-23 & Athole Point & 938 & 1.7 & -3.1 & 0.57 & 1707 & 79 & 74 \\
\hline TS-26 & Athole Point & 941 & 2.0 & -7.6 & 0.15 & 3000 & 140 & 226 \\
\hline TS-27 & Athole Point & 945 & 0.1 & -6.8 & 0.03 & 2118 & 26 & 795 \\
\hline TS-28 & Athole Point & 945 & 0.5 & -6.6 & 0.02 & 2294 & 28 & 434 \\
\hline TS-24 & Athole Point & 947 & 1.0 & -5.4 & 0.54 & 2164 & 182 & 130 \\
\hline TS-29 & Athole Point & 959 & 2.0 & -2.7 & 0.58 & 2255 & 52 & 93 \\
\hline TS-20 & Athole Point & 974 & 1.9 & -2.4 & 0.54 & 1381 & 32 & 74 \\
\hline TS-30 & Athole Point & 979 & 2.2 & -4.2 & 0.02 & 1977 & 10 & 659 \\
\hline TS-31 & Athole Point & 1001 & 0.2 & -4.8 & 0.02 & 2063 & 28 & 1133 \\
\hline TS-32 & Athole Point & 1007 & 1.7 & -5.7 & 0.02 & 1181 & 31 & 528 \\
\hline TS-33 & Athole Point & 1011 & 2.3 & -5.9 & 0.08 & 1958 & 74 & 733 \\
\hline TS-34 & Athole Point & 1011 & 1.3 & -5.2 & 0.02 & 1773 & 53 & 808 \\
\hline TS-35 & Athole Point & 1030 & 0.8 & -6.7 & 0.07 & 5549 & 355 & 822 \\
\hline TS-36 & Athole Point & 1037 & 2.7 & -5.5 & 0.03 & 3237 & 128 & 679 \\
\hline TS-37 & Athole Point & 1055 & 2.1 & -4.6 & 0.02 & 2295 & 38 & 880 \\
\hline TS-38 & Athole Point & 1070 & 2.3 & -5.7 & 0.06 & 4405 & 166 & 995 \\
\hline TS-39 & Athole Point & 1262 & -0.8 & -7.7 & 0.07 & 6676 & 376 & 1218 \\
\hline $\mathrm{TS}-40$ & Athole Point & 1296 & -0.2 & -5.5 & 0.38 & 8020 & 288 & 217 \\
\hline TS-41 & Athole Point & 1330 & -0.3 & -5.8 & 0.22 & 9158 & 434 & 674 \\
\hline $\mathrm{TS}-42$ & Athole Point & 1364 & 0.4 & -7.4 & 0.05 & 3387 & 158 & 2040 \\
\hline $\mathrm{TS}-43$ & Athole Point & 1398 & 0.8 & -6.9 & 0.02 & 3764 & 107 & 2364 \\
\hline TS-44 & Athole Point & 1432 & 0.7 & -5.3 & 0.03 & 3333 & 360 & 698 \\
\hline TS-45 & Athole Point & 1466 & 1.5 & -6.5 & 0.04 & 4000 & 179 & 557 \\
\hline
\end{tabular}

from addition of isotopic depleted fluids or temperature increases during burial, is typically large, given roughly equal fractions of oxygen in water and carbonate rock reservoirs (Banner and Hanson 1990).

Society Cliffs samples from Milne Inlet preserve a narrow range of $\delta^{13} \mathrm{C}(1 \%)$ accompanied by much greater variation in $\delta^{18} \mathrm{O}(10 \%)$, suggesting that primary $\delta^{13} \mathrm{C}$ was retained throughout diagenesis. Greater isotopic variability is recorded in White Bay samples, yet the absence of strong covariant trends is inconclusive to determine the origin of carbon isotopic variation. Five samples (cf. Tables 1, 2) were also tested for intrasample isotopic variation. Sample components differ in terms of depositional fabric, origin of carbonate, and the timing of carbonate formation. In contrast with other geochemical parameters, less than $0.3 \%$ variation in carbon isotopic composition was recorded between sample components, again suggesting first-order retention of primary carbon isotopic compositions.

Isotopic variation, however, can result from the reequilibration of carbonate with fluids during diagenesis or from the remineralization of isotopically light organic carbon during both depositional and early diagenetic processes. Whereas reequilibration results from decarbonation reactions or the release of isotopically light volatiles from organic carbon during metamorphism, isotopically light carbon can readily be made available for remineralization in nearsurface environments, either by the oxidation of sedimentary organic matter by $\mathrm{O}_{2}$-rich fluids, or by microbial degradation of organic matter.

The extent of postdepositional diagenesis can be approximated by examining geochemical trends that result from the significantly different chemistries of marine and diagenetic waters. Therefore, all samples were subjected to elemental analysis to determine $\mathrm{Fe}, \mathrm{Mn}$, and $\mathrm{Sr}$ abundances. In particular, the elemental ratio of $\mathrm{Mn}$ to $\mathrm{Sr}$ is commonly used as a diagenetic indicator; during meteoric diagenesis, $\mathrm{Mn}$ is commonly incorporated into sedimentary carbonates, whereas $\mathrm{Sr}$ is flushed from the carbonate lattice (Veizer 1983; Derry et al. 1992). Empirical evidence suggests that retention of primary carbon isotopic values is favored by the rapid stabilization of carbonate sediments during early marine diagenesis, and that carbonates with $\mathrm{Mn} / \mathrm{Sr}<10$ generally retain primary carbon isotopic compositions (Kaufman and Knoll 1995).

Figure 5 shows Mn/Sr ratios of Bylot Supergroup carbonate rocks plotted against $\delta^{18} \mathrm{O}$. Mn/Sr ratios of all Milne Inlet and Tremblay Sound samples are less than 4, well within the range of accepted values for "little-altered" carbonates. With the exception of a single sample, $\mathrm{Mn} / \mathrm{Sr}$ ratios of White Bay and Mala River samples are also much less than 10. Samples 
Table 2. Isotopic and elemental compositions, White Bay and Mala River.

\begin{tabular}{|c|c|c|c|c|c|c|c|c|}
\hline $\begin{array}{l}\text { Sample } \\
\text { No. }{ }^{a}\end{array}$ & Formation & $\begin{array}{l}\text { Height } \\
\text { (m) }\end{array}$ & $\begin{array}{l}\delta^{13} \mathrm{C} \\
(\% \circ \mathrm{PDB})\end{array}$ & $\begin{array}{l}\delta^{18} \mathrm{O} \\
(\% \circ \mathrm{PDB})^{b}\end{array}$ & $\mathrm{Mg} / \mathrm{Ca}$ & $\begin{array}{l}\mathrm{Fe} \\
(\mathrm{ppm})\end{array}$ & $\begin{array}{l}\mathrm{Mn} \\
(\mathrm{ppm})\end{array}$ & $\begin{array}{l}\mathrm{Sr} \\
(\mathrm{ppm})\end{array}$ \\
\hline WB-1 & Society Cliffs & 186 & 3.7 & -3.3 & 0.55 & 824 & 104 & 43 \\
\hline WB-2 & Society Cliffs & 199 & 3.5 & -4.5 & 0.54 & 950 & 95 & 56 \\
\hline WB-3 & Society Cliffs & 208 & 3.3 & -2.4 & 0.55 & 1039 & 67 & 64 \\
\hline WB-4 & Society Cliffs & 215 & 2.8 & -2.4 & 0.57 & 1000 & 62 & 67 \\
\hline WB-5 & Society Cliffs & 223 & 2.6 & -1.0 & 0.59 & 1125 & 192 & 38 \\
\hline WB-6 & Society Cliffs & 232 & 3.0 & -4.6 & 0.55 & 1042 & 170 & 47 \\
\hline WB-8 & Society Cliffs & 267 & 3.7 & -5.0 & 0.56 & 885 & 115 & 41 \\
\hline WB-9 & Society Cliffs & 278 & 2.9 & -3.8 & 0.57 & 1093 & 244 & 31 \\
\hline WB-10 & Society Cliffs & 303 & 2.7 & -2.4 & 0.53 & 938 & 219 & 49 \\
\hline WB-11 & Society Cliffs & 321 & 2.4 & -3.3 & 0.54 & 1081 & 241 & 48 \\
\hline WB-12a & Society Cliffs & 331 & 2.6 & -3.5 & 0.55 & 667 & 179 & 26 \\
\hline WB-12b & Society Cliffs & 331 & 2.7 & -3.4 & 0.58 & 1243 & 461 & 61 \\
\hline WB-13 & Society Cliffs & 344 & 2.0 & -3.1 & 0.58 & 1226 & 277 & 35 \\
\hline WB-14 & Society Cliffs & 345 & 2.7 & -0.7 & 0.56 & 1143 & 339 & 39 \\
\hline WB-15 & Society Cliffs & 372 & 2.9 & -1.8 & 0.58 & 1288 & 140 & 69 \\
\hline WB-16 & Society Cliffs & 403 & 2.9 & -2.8 & 0.58 & 1417 & 545 & 66 \\
\hline WB-17 & Society Cliffs & 422 & 3.0 & -3.0 & 0.57 & 3250 & 471 & 50 \\
\hline MR-2 & Victor Bay & 677 & 0.6 & -5.0 & 0.49 & 2733 & 149 & 116 \\
\hline MR-3a & Victor Bay & 699 & 0.2 & -4.6 & 0.20 & 2063 & 97 & 313 \\
\hline MR-3b & Victor Bay & 699 & 0.4 & -5.7 & 0.02 & 3000 & 41 & 725 \\
\hline MR-4 & Victor Bay & 771 & 1.1 & -4.5 & 0.46 & 2816 & 104 & 155 \\
\hline MR-5 & Victor Bay & 847 & 0.4 & -2.4 & 0.47 & 3548 & 187 & 126 \\
\hline MR-7 & Victor Bay & 872 & 0.9 & -3.2 & 0.31 & 1868 & 117 & 130 \\
\hline MR-6 & Victor Bay & 882 & 0.7 & -4.1 & 0.55 & 1217 & 106 & 318 \\
\hline MR-8 & Victor Bay & 887 & 0.6 & -5.6 & 0.02 & 4061 & 59 & 705 \\
\hline MR-9a & Victor Bay & 900 & 1.1 & -3.9 & 0.24 & 3167 & 152 & 238 \\
\hline MR-9b & Victor Bay & 900 & 1.4 & -5.6 & 0.05 & 3221 & 57 & 595 \\
\hline MR-10 & Victor Bay & 900 & 1.4 & -4.2 & 0.18 & 3293 & 87 & 754 \\
\hline MR-12 & Victor Bay & 964 & 1.8 & -5.6 & 0.05 & 1119 & 175 & 1357 \\
\hline MR-13 & Athole Point & 1065 & 1.8 & -6.0 & 0.05 & 1923 & 73 & 155 \\
\hline MR-14 & Athole Point & 1131 & 2.9 & -5.3 & 0.05 & 2683 & 117 & 838 \\
\hline MR-15 & Athole Point & 1295 & 1.0 & -4.3 & 0.14 & 5583 & 319 & 230 \\
\hline MR-16 & Athole Point & 1442 & 1.1 & -4.1 & 0.22 & 5060 & 272 & 457 \\
\hline
\end{tabular}

${ }^{a}$ WB, White Bay; MR, Mala River.

${ }^{b}$ Dolomites are corrected for phosphoric acid fractionation at $90^{\circ} \mathrm{C}$ during processing.

from the Society Cliffs Formation, however, are consistently enriched in $\mathrm{Mn} / \mathrm{Sr}$ ratios relative to Victor Bay and Athole Point values. This trend may be misleading in that actual abundances of $\mathrm{Mn}$ are similar for the three formations (cf. Tables 1, 2). The relative enrichment of $\mathrm{Mn} / \mathrm{Sr}$ ratios is likely a consequence of the low $\mathrm{Sr}$ concentration in dolomitic samples relative to calcareous samples. Low $\mathrm{Sr}$ concentrations are common in dolomitic rocks; the size of the $\mathrm{Sr}$ ion imparts a strong preference for the Ca sites in carbonate rocks (Pierson 1981), and consequently Sr is expelled from the crystal during dolomitization. Sr values fall significantly with increasing $\mathrm{Mg}$ content (Sathyanarayan et al. 1987; Carpenter et al. 1991) and suggest that rapid precipitation of dolomitic phases may partition strongly against $\mathrm{Sr}$ ions. Enriched $\mathrm{Mn} / \mathrm{Sr}$ ratios may therefore result, in part, from the early diagenetic dolomitization of the Society Cliffs Formation.

Because dolomitization has been considered a potential agent for carbon isotopic alteration, the chemical signature of dolomites relative to limestones in the same succession is important when evaluating diagenetic trends. In Neoproterozoic and Early Cambrian successions that have been extensively sampled for isotopic analysis, empirical observation shows that $\delta^{13} \mathrm{C}$ variation of both limestones and dolomites accurately trace the same pattern of secular variation (Knoll et al. 1986; Narbonne et al. 1994; Kaufman and Knoll 1995; Pelechaty et al. 1996), although minor variation in isotopic composition may be observed (Pelechaty et al. 1996). Possible factors for observed variation in lithologic and isotopic composition are discussed in depth by Pelechaty et al. (1996) and include postdepositional dolomitization from fluids of differing isotopic composition, equilibrium fraction between limestone and dolostone phases (Sheppard and Schwarcz 1970), and depositional variation in fluid composition reflected in synsedimentary dolomite formation. In the Victor Bay Formation, subtidal limestone and laterally equivalent peritidal dolostone do not reveal any obvious difference in $\delta^{13} \mathrm{C}$ (Fig. 6). That limestones and dolostones track identical chemostratigraphic trends without significant offset suggests either that the pre- 
Fig. 4. $\delta^{13} \mathrm{C}$ vs. $\delta^{18} \mathrm{O}$ from Bylot Supergroup carbonate rocks collected at (A) Milne Inlet and Tremblay Sound, and (B) White Bay and Mala River.

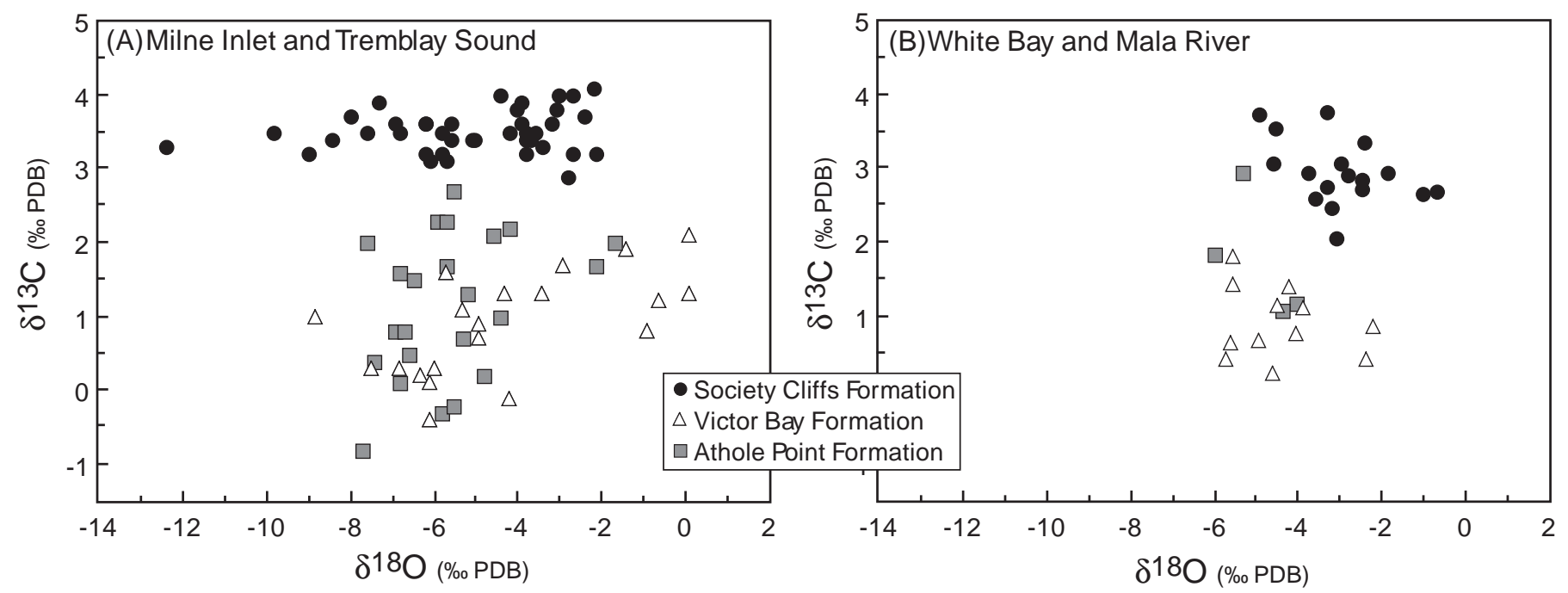

Fig. 5. Mn/Sr ratios of Bylot Supergroup carbonate rocks collected at (A) Milne Inlet and Tremblay Sound, and (B) White Bay and Mala River. Note the difference in vertical scale between (A) and (B).

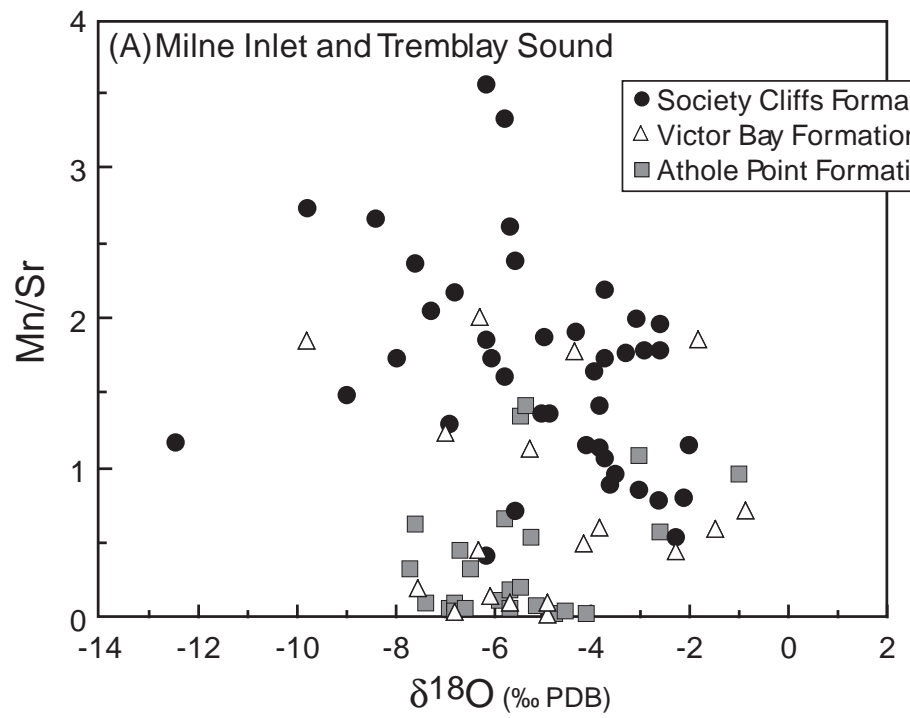

cursor limestone was the primary source for dolomitic carbon, or that marine and dolomitic fluids were of similar composition.

Comparison of $\delta^{18} \mathrm{O}$ between limestones and dolostones may help resolve these possibilities. Excessively light oxygen isotopic values commonly result from isotopic exchange with meteoric waters or equilibration at elevated temperatures during burial. Such postdepositional processes typically involve large volumes of fluid exchange and commonly result in homogenization of isotopic compositions (Land 1980). Bylot Supergroup carbonates have an average $\delta^{18} \mathrm{O}$ of approximately $-5.2 \%$ (cf. Fig. 4). In subtidal to intertidal sections, both Society Cliffs dolostones and Victor Bay Athole Point limestones show a considerable spread of values, from -0.9 to $-12.4 \%$, whereas $\delta^{18} \mathrm{O}$ values for intertidal to supratidal, predominantly dolomitic sections show a much narrower range of values. Isotopic values of $\delta^{18} \mathrm{O}$ in sedimentary dolomites commonly show a more restricted range

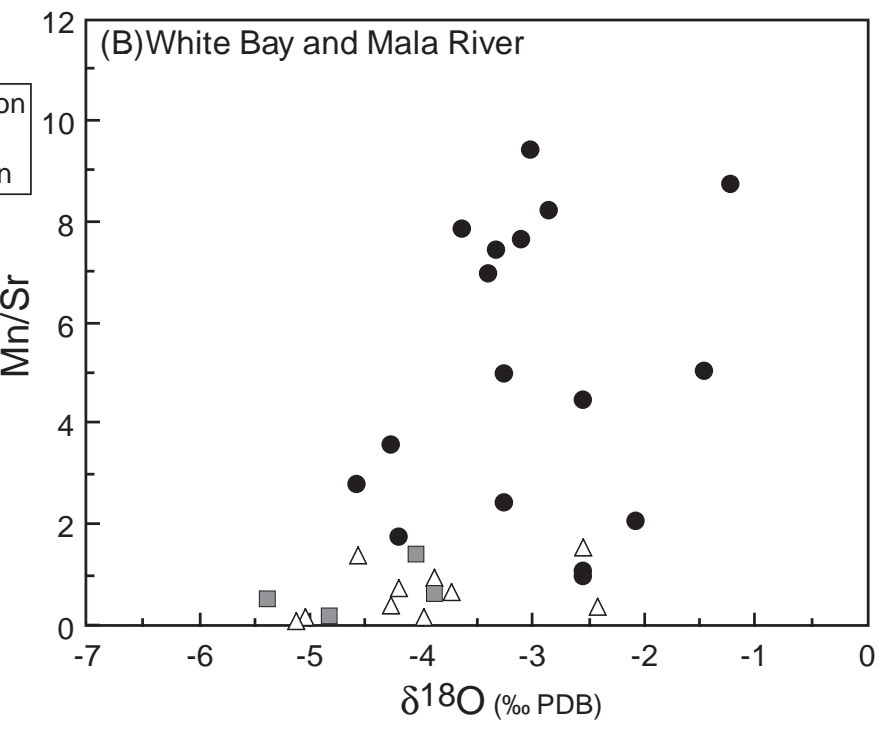

than limestones in the same unit (cf. Buick et al. 1995), and the greater isotopic variability in subtidal limestones is consistent with the suggestion that early dolomitization of sedimentary carbonates may actually aid in the retention of primary geochemical signatures by restricting pore space and limiting subsequent fluid interaction (Fairchild et al. 1990).

Average $\delta^{18} \mathrm{O}$ values approximately $-6 \%$ are not unusual for Proterozoic sediments (Veizer and Hoefs 1976; Veizer et al. 1992). It has been proposed that such values record stabilization in isotopically light marine waters, whereas heavier $\delta^{18} \mathrm{O}$ values reflect locally evaporitic conditions (Burdett et al. 1990). Within Society Cliffs dolostones, $\delta^{18} \mathrm{O}$ shows a facies-dependent variation consistent with observed paleogeographical evidence, suggesting that heavy oxygen isotopic values are a primary feature related to the paleogeographic restriction and evaporative enrichment of shallow marine waters (Kah 1997). Patterns of oxygen isoto- 
Fig. 6. Stratigraphic variation in $\delta^{13} \mathrm{C}$ from Society Cliffs, Victor Bay, and Athole Point formations. Sections are compiled into deeper and shallower water sections. On the left is a composite of laterally adjacent sections measured at Milne Inlet (MI; peritidal Society Cliffs Formation) and Tremblay Sound (TS; dominantly subtidal Victor Bay and Athole Point formations). On the right is a composite of sections measured at White Bay (WB; peritidal Society Cliffs Formation) and Mala River (MR; peritidal Victor Bay and dominantly subtidal Athole Point formations).

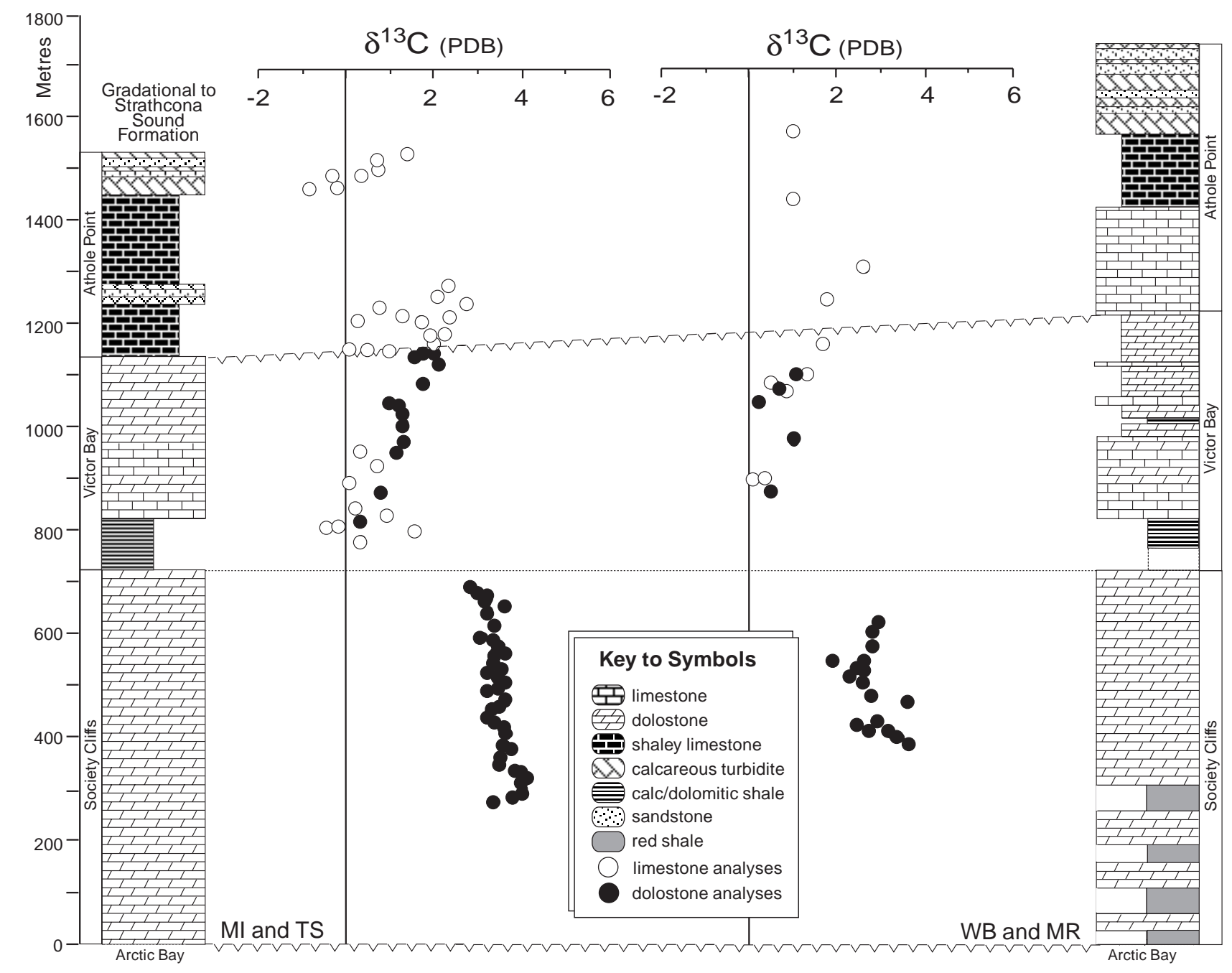

pic variation are also consistent with petrographic evidence of syndepositional, fabric-retentive dolomite formation, thereby suggesting that isotopic compositions imparted during early dolomitization were affected primarily by depositional variation in seawater compositions (Kah 1997).

\section{Stratigraphic variation in $\delta^{13} \mathbf{C}$}

Plotting $\delta^{13} \mathrm{C}$ versus stratigraphic height for Society Cliffs, Victor Bay, and Athole Point formations reveals a tripartite architecture: an isotopically heavy plateau $(\sim 3.5 \%$ ) followed by two positive excursions to $\sim 3 \%$ o that arise from isotopic values near $-1 \%$ o (Fig. 6). The Society Cliffs Formation at Milne Inlet contains the most positive $\delta^{13} \mathrm{C}$ ratios of the succession $(+2.9$ to $+4.1 \%$ ) . This isotopic plateau is largely invariant, showing only a small, $1 \%$ o decrease in $\delta^{13} \mathrm{C}$ upwards through the section. Commencement of the Victor Bay Formation is marked by regional deposition of pyritic, siliciclastic to calcareous black shale. Carbonate interbeds near the top of this interval are marked by $\delta^{13} \mathrm{C}$ values near
$0 \%$, followed by a continuous rise in $\delta^{13} \mathrm{C}$ upwards through the formation, reaching a maximum of $+1.7 \%$. This broad positive excursion continues into the lower Athole Point Formation to a high of $+2.7 \%$. Superimposed on this broader positive excursion, limestones of the lower Victor Bay Formation exhibit a rapid $1 \%$ alternation, and four points $(\mathrm{TS}-27=+0.1 \%$; $\mathrm{TS}-28=+0.5 \%$; $\mathrm{TS}-31=+0.2 \%$; TS-35 $=+0.8 \%$ ) preserve isotopic values significantly lighter than the general range of values. There is no reason to believe that these samples have been altered to any greater extent, suggesting that this variability may be primary. Higher in the section, the middle Athole Point Formation is again marked by isotopically light $\delta^{13} \mathrm{C}$ values and a strong positive excursion in the overlying strata from a basal value of $-0.8 \%$ o to an uppermost value of $+1.5 \%$.

Laterally equivalent strata at White Bay and Mala River were more sparsely sampled, and as such have a significantly lower chemostratigraphic resolution. Nonetheless, sections display a strikingly similar pattern of $\delta^{13} \mathrm{C}$ changes. 
The Society Cliffs Formation shows a generally decreasing trend from $+3.7 \%$ o to approximately $+3.0 \%$. Victor Bay samples mimic both the positive trend and the absolute magnitude of the isotopic excursion observed in more basinal sections. With only four samples from the Athole Point Formation, isotopic trends cannot be determined with certainty; however, observed positive values $(+2.9 \%$ ) are similar to the most positive values $(+2.7 \%$ ) recorded in equivalent stratigraphic positions at Tremblay Sound.

\section{Depositional variation in $\delta^{\mathbf{1 3}} \mathbf{C}$}

Carbonate rocks from the Bylot Supergroup show distinct trends with stratigraphic height, and isotopic values do not appear to have been strongly affected by postdepositional diagenetic processes. Many of the processes, however, likely to have affected carbon isotopic compositions in the rock record result from isotopic exchange between organic and inorganic carbon reservoirs at the time of carbonate formation and during earliest marine diagenesis. Routine tests for postdepositional alteration associated with meteoric or hydrothermal fluid flow cannot address the affects of biogenic processes on the isotopic composition of carbonate rocks.

In the oceans, both chemical and biological processes utilize the same dissolved inorganic carbon (DIC) source. Reactions associated with biological metabolism show large kinetic isotope effects, resulting in a $-25 \%$ o to $-30 \%$ depletion of organic carbon relative to carbonate derived from the same source (Schidlowski et al. 1983). Whereas photosynthetic uptake of isotopically light carbon results in a relative enrichment in ${ }^{13} \mathrm{C}$ in the surrounding oceans, isotopically light carbon is returned to the dissolved inorganic carbon pool during biological respiration and processes of organic degradation. Heterogeneities in the isotopic composition of dissolved inorganic carbon can therefore arise whenever the processes of organic production and degradation in any given water mass are not in equilibrium. Depositional variation typically results from either depth-dependent oceanographic gradients (Broecker and Peng 1982) or microbial metabolism of sedimentary organic matter (Schidlowski et al. 1983; Coleman 1993).

Exchange between carbonate carbon and organic carbon reservoirs can be evaluated by examining both the composition of organic carbon $\left(\delta^{13} \mathrm{C}_{\text {org }}\right)$ and the difference between carbonate carbon and organic carbon isotopic signals $(\Delta \delta=$ $\delta^{13} \mathrm{C}_{\text {carb }}-\delta^{13} \mathrm{C}_{\text {org }}$ ). If biological fractionation does not change through a stratigraphic section, $\delta^{13} \mathrm{C}$ and $\delta^{13} \mathrm{C}_{\text {org }}$ should track together (i.e., a constant $\Delta \delta$; Knoll et al. 1986; Hayes et al. 1989). Variation in $\Delta \delta$ arises with exchange between inorganic and organic carbon reservoirs. Degradation of primary organic matter releases isotopically light carbon and typically results in a subsequent depletion of carbonate phases. Likewise, kinetic isotope effects govern the preferential uptake of ${ }^{12} \mathrm{C}$ during heterotrophic activity, resulting in an isotopic enrichment of residual organic matter. Therefore, a decline in $\Delta \delta$, correlated with a decline in carbonate $\delta^{13} \mathrm{C}$, can be interpreted as the result of isotopic exchange between organic and inorganic carbon reservoirs.

Organic carbon analyses were undertaken for a selected suite of Society Cliffs samples to assess potential varia- tion related to isotopic exchange reactions (Table 3). $\delta^{13} \mathrm{C}_{\text {org }}$ for the majority of samples lies between $-30 \%$ and $-26 \%$ and show an increased fractionation with higher total organic carbon (TOC) (Fig. 7). Except at the lowest TOC contents, $\Delta \delta$ is $29 \pm 1 \%$. These values compare favorably to those commonly observed in Proterozoic successions (Knoll et al. 1986 1995; Des Marais et al. 1992) and suggest that isotopic compositions of carbonate and organic carbon have not been strongly affected by post-depositional processes.

Paired carbonate and organic carbon analyses, however, show minor variation that may elucidate local depositional variation in $\delta^{13} \mathrm{C}$. Microbial dolostone from White Bay has carbon isotopic values generally $\sim 1 \%$, and as much as nearly $2 \%$, lighter than corresponding oolitic dolostone from Milne Inlet (Tables 1, 2; Fig. 4). Similar isotopic depletions of $\sim 1.0 \%$ are observed in microbially laminated dolostone from the Nanisivik region (Ghazban et al. 1992). Petrographic evidence of White Bay carbonates indicates that carbonate precipitation occurred in situ within mats during microbial degradation (Kah and Knoll 1996). These small, 1-2\%o depletions therefore likely result from carbonate precipitation in the presence of isotopically light carbon released during mat respiration or heterotrophic degradation.

A corollary to this hypothesis suggests that sedimentary successions consisting primarily of carbonate precipitated within the water column, i.e., not within close proximity to concentrated products of organic degradation, should more accurately reflect the isotopic compositions of marine waters. Six samples from the Society Cliffs Formation which formed either in the water column, as oolites, or on the sea floor, as encrusting precipitates, have low TOC and enriched isotopic compositions of both organic and carbonate carbon (Table 3; Fig. 7). Although the association of isotopically enriched organic matter and low organic content may reflect the relative ease of contamination at low organic contents, most modern contaminants would be isotopically depleted with respect to ${ }^{13} \mathrm{C}$. Another possibility is that isotopic compositions reflect the isotopically enriched nature of highly degraded particulate organic matter, as would be expected present in low concentrations in the water column.

\section{Secular variation in $\delta^{13} \mathrm{C}$}

The carbon isotopic profile for Bylot Supergroup carbonates shows primary $\delta^{13} \mathrm{C}$ values near $3-3.5 \%$, and contains two robust negative excursions of up to $4 \%$ (Fig. 6). Local isotopic variation related to remineralization of sedimentary organic material is unlikely to have produced such large variations: assuming an original $\delta^{13} \mathrm{C}_{\mathrm{carb}}$ of $+3.5 \%$ and an original $\delta^{13} \mathrm{C}_{\text {org }}$ of $-30 \%$, mass balance requires that at least $9 \%$ of the carbonate would have to originate from organic carbon to produce the observed $4 \%$ o negative shifts. Rather, the observed stratigraphic excursions are likely to result from sampling true variation in the primary carbon isotopic composition of marine waters. The correspondence of the lowermost carbon isotopic excursion with a major flooding surface and facies change from peritidal carbonate to subtidal shale (Fig. 6) suggests the possibility of direct measurement of a depth-related isotopic gradient. Several observations, however, suggest that this scenario is unlikely. First, the observed $4 \%$ o isotopic shifts are of greater magnitude 
Table 3. Society Cliffs Formation organic carbon analyses.

\begin{tabular}{|c|c|c|c|c|c|c|}
\hline Sample No. & Lithology & $\begin{array}{l}\text { TOC } \\
(\mathrm{mg} / \mathrm{g})\end{array}$ & $\begin{array}{l}\delta^{13} \mathrm{C}_{\mathrm{carb}} \\
(\% \circ \mathrm{PDB})\end{array}$ & $\begin{array}{l}\delta^{18} \mathrm{O} \\
(\% \circ \mathrm{PDB})^{a}\end{array}$ & $\begin{array}{l}\delta^{13} \mathrm{C}_{\text {org }} \\
(\% o)\end{array}$ & $\begin{array}{l}\Delta \delta \\
(\% o)\end{array}$ \\
\hline A1-5829 & Dolomite & 0.90 & - & - & -25.8 & - \\
\hline A3-2629 & Dolomite & 1.50 & 1.8 & -3.8 & -29.3 & 31.1 \\
\hline A3-5387 & Dolomite & 0.21 & 3.0 & -5.8 & -19.4 & 22.4 \\
\hline EWB & Dolomite & 0.37 & - & - & -25.6 & - \\
\hline ТH3 & Dolomite & 0.95 & 2.8 & -2.7 & -27.2 & 30.0 \\
\hline TP-10920a & Dolomite & 0.18 & 3.3 & -4.6 & -22.7 & 26.0 \\
\hline TP-10920b & Dolomite & 0.20 & 3.4 & -5.7 & -22.7 & 26.1 \\
\hline TP-24210 & Dolomite & 0.17 & 3.0 & -5.8 & -20.5 & 23.5 \\
\hline TY-18025 & Dolomite & 1.51 & 3.1 & -3.5 & -27.2 & 30.3 \\
\hline TY-2165c & Dolomite & 0.36 & 3.4 & -3.1 & -26.8 & 30.2 \\
\hline WB-16660 & Dolomite & 0.30 & 2.3 & -3.8 & -25.2 & 27.5 \\
\hline WB-29570 & Dolomite & 0.70 & 2.6 & -3.7 & -26.9 & 29.5 \\
\hline WB-48a & Dolomite & 2.36 & 2.3 & -4.1 & -29.3 & 31.6 \\
\hline WB-94-12 & Dolomite & 0.82 & 2.8 & -2.5 & -28.0 & 30.8 \\
\hline WB-94-17 & Dolomite & 0.92 & 2.9 & -2.6 & -27.6 & 30.5 \\
\hline A2-37855 & Dolomite & 0.52 & 2.3 & -3.9 & -27.2 & 29.5 \\
\hline A2-37855 & Chert & 1.02 & - & - & -30.2 & - \\
\hline TY-20245 & Dolomite & 0.47 & 3.4 & -3.6 & -25.0 & 28.4 \\
\hline TY-20245 & Chert & 0.32 & - & -2.6 & -21.7 & - \\
\hline TY2-14988 & Dolomite & 0.78 & 1.2 & -4.5 & -26.9 & 28.1 \\
\hline TY2-14988 & Chert & 1.36 & - & - & -26.6 & - \\
\hline WB-18034 & Dolomite & 2.58 & 1.7 & -2.3 & -29.4 & 31.1 \\
\hline WB-18034 & Chert & 1.54 & - & -1.9 & -27.8 & - \\
\hline WB-8675 & Dolomite & 2.95 & 1.7 & -2.7 & -28.8 & 30.5 \\
\hline WB-8675 & Chert & 1.83 & - & -1.0 & -28.3 & - \\
\hline WB-5 & Chert & 1.94 & - & -1.7 & -28.9 & - \\
\hline
\end{tabular}

${ }^{a} \delta^{18} \mathrm{O}$ dolomite corrected for phosphoric acid fractionation at $90^{\circ} \mathrm{C}$.

Fig. 7. $\delta^{13} \mathrm{C}_{\text {org }}$ vs. total organic carbon (TOC) of dolomitic and chert samples, Society Cliffs Formation. See text for explanation.

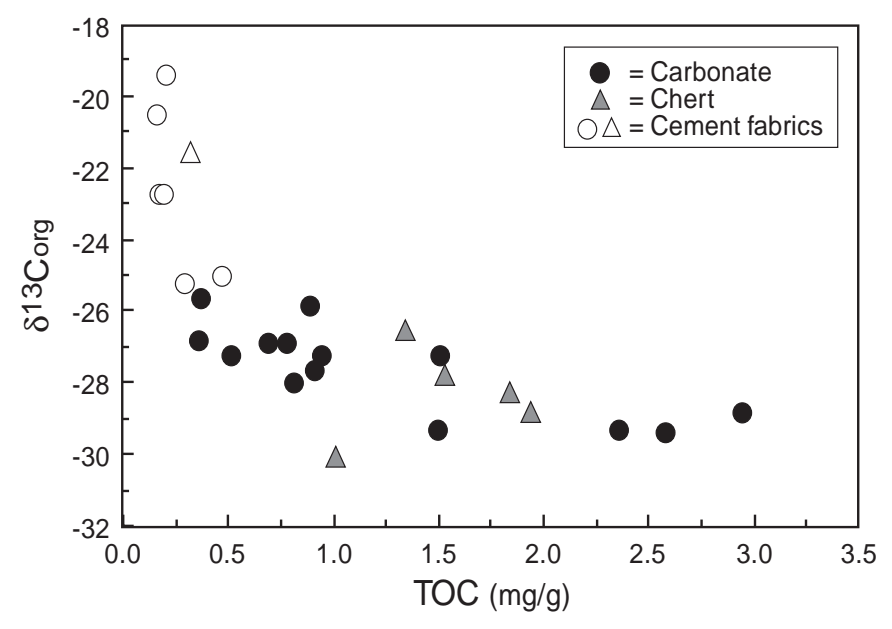

than isotopic gradients typically observed in mixed-water regimes; $\delta^{13} \mathrm{C}$ gradients in modern oceans are typically less than $\sim 2 \%$ o (Broecker and Peng 1982). Second, as facies shift back to predominantly shallow-water carbonate lithologies, values do not return to pre-excursion values. In fact, positive values of $\sim 3 \%$ in the lower Athole Point Formation occur in deep subtidal carbonates, unlike the peritidal carbonates of the Society Cliffs formation. Finally, strikingly similar isotopic profiles observed in coeval subtidal and peritidal carbonates of the upper Victor Bay Formation suggest that isotopic depth gradients contribute little to the observed pattern of isotopic variation.

Because stratigraphic shifts do not appear to be associated with either depositional environment or organic carbon content, they are interpreted as representing secular variation in marine surface waters. Short-lived negative carbon isotopic excursions in marine carbonate rocks have been attributed either to the input of isotopically light carbon into the ocean-atmosphere system (Dickens et al. 1995; Bralower et al. 1997), or to the rapid mixing of isotopically light deep waters with the surface ocean (Kaufman et al. 1991). During at least part of the Proterozoic, water-body stratification and diminished export of inorganic carbon into the deep ocean could have resulted in increased surface-to-deep gradients (Grotzinger and Knoll 1995; Bartley et al. 1998). Ventilation of deep waters has been hypothesized to occur with enhanced circulation (Bartley et al. 1998), possibly related to glacial episodes (Knoll et al. 1996; Kaufman et al. 1997). No evidence for glaciation, however, has been reported from the Mesoproterozoic. Continental rifting (Bond et al. 1984), or marine transgression (Saltzman et al. 1995), may not be ruled out as mechanisms that may have affected stratigraphic variation in $\delta^{13} \mathrm{C}$ via changing burial fluxes of organic and carbonate carbon. 
Table 4. Isotopic and elemental compositions of Hunting Formation carbonates, Somerset Island.

\begin{tabular}{|c|c|c|c|c|c|c|c|}
\hline Sample No. & $\begin{array}{l}\text { Height } \\
\text { (m) }\end{array}$ & $\begin{array}{l}\delta^{13} \mathrm{C} \\
(\% \circ \mathrm{PDB})\end{array}$ & $\begin{array}{l}\delta^{18} \mathrm{O} \\
(\% \circ \mathrm{PDB})^{a}\end{array}$ & $\mathrm{Mg} / \mathrm{Ca}$ & $\begin{array}{l}\mathrm{Fe} \\
(\mathrm{ppm})\end{array}$ & $\begin{array}{l}\mathrm{Mn} \\
(\mathrm{ppm})^{b}\end{array}$ & $\begin{array}{l}\mathrm{Sr} \\
(\mathrm{ppm})\end{array}$ \\
\hline HU-HY-O & 0 & 1.4 & -3.6 & 0.68 & nd & 539 & 51 \\
\hline 87-HS-25 & 8 & 0.3 & -3.9 & 0.70 & nd & 983 & 52 \\
\hline HU-0070 & 21 & 1.8 & -2.3 & 0.70 & nd & 79 & 51 \\
\hline HU-0351 & 107 & 2.2 & -4.8 & 0.73 & nd & 65 & 37 \\
\hline HU-0575 & 175 & 2.3 & -4.8 & 0.72 & nd & 78 & 28 \\
\hline HU-0676 & 206 & 2.7 & -3.8 & 0.73 & nd & 37 & 43 \\
\hline HU-0755 & 230 & 3.0 & -4.0 & 0.71 & nd & 74 & 27 \\
\hline HU-0845 & 258 & 2.0 & -4.8 & 0.71 & nd & 98 & 30 \\
\hline HU-0935 & 285 & 2.6 & -5.4 & 0.71 & nd & 132 & 57 \\
\hline HU-0950 & 290 & 2.3 & -4.3 & 0.72 & nd & 104 & 29 \\
\hline HU-0955-1 & 291 & 2.2 & -4.5 & 0.69 & nd & 90 & 26 \\
\hline HU-0955-2 & 291 & 2.4 & -4.8 & 0.78 & nd & 185 & 41 \\
\hline HU-1065 & 325 & 0.8 & -2.1 & 0.73 & nd & 26 & 38 \\
\hline HU-1275 & 389 & 1.8 & -4.8 & 0.72 & nd & 101 & 26 \\
\hline HU-1375 & 419 & 2.5 & -5.5 & 0.73 & nd & 65 & 27 \\
\hline HU-1465 & 446 & 3.0 & -4.8 & 0.74 & nd & 33 & 24 \\
\hline HU-1570 & 478 & 3.2 & -5.4 & 0.76 & nd & 96 & 26 \\
\hline HU-1671 & 509 & 2.5 & -4.2 & 0.73 & nd & 36 & 47 \\
\hline HU-1783 & 544 & 2.6 & -3.1 & 0.73 & nd & 98 & 52 \\
\hline HU-1873 & 571 & 2.4 & -13.9 & 0.72 & nd & 84 & 51 \\
\hline
\end{tabular}

\section{Implications for regional correlation}

Despite a relatively limited range of values and poor control on the timing of excursions, isotopic variation preserved in the Bylot Supergroup appears to faithfully record the temporal isotopic variation of marine surface waters. These variations may therefore provide a means, independent of lithology and biostratigraphy, of testing proposed regional stratigraphic relationships. Of particular interest are the relative age relationships among the Bylot Supergroup and other potentially correlative successions in northern Laurentia: the Hunting Formation (Somerset Island), Narssârssuk Formation (Greenland), and Shaler Supergroup (Victoria Island).

Young $(1979,1981)$ divided the Proterozoic sedimentary record of Laurentia into three broadly correlative successions based on regional unconformities, deformational events, and sparse radiometric data. The late Mesoproterozoic to Neoproterozoic Succession B (Young 1979) is defined by its position stratigraphically above $\sim 1270 \mathrm{Ma}$ mafic rocks of the widespread Mackenzie igneous event, and by its intrusive relationship with mafic dikes of the $\sim 723 \mathrm{Ma}$ Franklin event. In northwestern Laurentia, Succession B is represented by the Shaler Supergroup (Rainbird et al. 1994), which lies unconformably above Mackenzie-age flood basalts of the Coppermine River Group and is both intruded by Franklinian intrusive rocks and overlain by coeval Natkusiaq flood basalts (Young 1981). Following this scheme, sedimentary rocks of the Bylot Supergroup, Aston and Hunting formations (Somerset Island), and Thule Supergroup (northwest Greenland) are often considered, despite >500 Ma age range provided by radiometric data, correlatives of the
Shaler Supergroup of the Amundsen Embayment and equivalents across northern and western Laurentia (Young 1981).

Sedimentary successions of Baffin Island, Somerset Island, and Greenland have long been presumed coeval, based on stratigraphic similarity (Jackson and Ianelli 1981), yet controversy remains as to the depositional relationships among the basins. The basins may have evolved initially as separate depocenters (Jackson and Iannelli 1981; Dawes 1997) that were perhaps interconnected at a later stage of development (Jackson and Iannelli 1981; Jackson 1986). Sequence stratigraphic analysis indicates that, at the time deposition, the extremely low topographic profile of the Society Cliffs Formation resulted in broadly synchronous deposition over and aerial extent far exceeding present-day outcrop (Kah 1997). If this interpretation is correct, carbonate rocks of the Hunting and Narssârssuk formations may indeed be genetically related to those preserved in the Bylot Supergroup (cf. Jackson 1986; Hofmann and Jackson 1996) and should therefore record similar isotopic profiles.

The Hunting Formation consists of $\sim 700 \mathrm{~m}$ of peritidal carbonate and siliciclastic rocks preserved in a small basin on northwestern Somerset Island (Tuke et al. 1966; Dixon 1974). Lowermost strata of the Hunting Formation consist of coarse-grained sandstone which grades rapidly into variegated mudstone and siltstone interbedded with microbial dolostone (Reinson et al. 1976), sea-floor precipitates, and abundant chert. The upper $500 \mathrm{~m}$ are dominated by cyclic units of grainstone and stromatolitic dolostone that contain evidence of episodic subaerial exposure (R.H. Rainbird, unpublished data). Whereas Hunting Formation lithologies and depositional environments are similar to those preserved in 
Table 5. Isotopic compositions of Narssârssuk Formation carbonates, Thule Group, Greenland.

\begin{tabular}{|c|c|c|c|c|c|c|}
\hline Sample No. & Member & $\begin{array}{l}\text { TOC } \\
(\mathrm{mg} / \mathrm{g})\end{array}$ & $\delta^{13} \mathrm{C}_{\mathrm{org}}(\% o)$ & $\begin{array}{l}\delta^{13} \mathrm{C}_{\mathrm{carb}} \\
(\% \circ \mathrm{PDB})\end{array}$ & $\begin{array}{l}\delta^{18} \mathrm{O} \\
(\% \circ \mathrm{PDB})^{a}\end{array}$ & $\begin{array}{l}\Delta \delta \\
(\% 0)\end{array}$ \\
\hline KS-78-7-4 & Lower Red & 0.9 & -31.5 & 1.8 & -3.5 & 33.3 \\
\hline KS-78-7-1 & Lower Red & 0.6 & -30.6 & 1.4 & -5.4 & 32.0 \\
\hline KS-78-9-6 & Lower Red & 0.6 & -31.3 & 2.0 & -6.3 & 33.3 \\
\hline KS-78-12 & Aorfêrneq & 0.8 & -29.1 & 0.9 & 1.7 & 30.0 \\
\hline KS-78-13 & Aorfêrneq & 1.0 & -30.5 & 2.2 & -0.6 & 32.7 \\
\hline KS-78-18 & Aorfêrneq & 1.2 & -31.2 & 1.4 & -1.6 & 32.6 \\
\hline KS-78-19 & Aorfêrneq & 0.1 & -28.7 & 0.6 & -5.7 & 29.4 \\
\hline KS-78-25 & Aorfêrneq & 0.3 & -29.8 & 0.7 & -3.0 & 30.5 \\
\hline KS-78-11-3 & Upper Red & 0.1 & -27.5 & 2.7 & -0.2 & 30.2 \\
\hline KS-78-11-4 & Upper Red & 0.2 & -27.3 & 1.6 & 0.9 & 28.9 \\
\hline KS-78-11-9 & Upper Red & 0.1 & -26.6 & 2.2 & 1.8 & 28.8 \\
\hline KS-78-11-8 & Upper Red & 0.1 & -27.4 & 2.3 & 0.5 & 29.7 \\
\hline
\end{tabular}

Note: All samples are whole-rock samples, collected in 1978 by A.H. Knoll and P. Strother.

${ }^{a}$ Dolomites were corrected for phosphoric acid fractionation at $90^{\circ} \mathrm{C}$ during processing.

the Society Cliffs Formation, similar lithologies are absent in both Victor Bay and Athole Point formations. Twenty samples were analyzed from Hunting Formation strata (Table 4). Petrographic preservation, nonluminescent character, and generally low Mn abundances indicate that Hunting Formation samples are unlikely to have undergone extensive postdepositional alteration. After a rapid rise from values near $0 \%$, carbon isotopic values remain constant near $+2.2 \%$. The absence in Hunting carbonates of large (up to $4 \%$ o) stratigraphic shifts in $\delta^{13} \mathrm{C}$ suggests that it was not deposited coevally with Victor Bay or Athole Point strata. Observed isotopic invariance, though inconclusive, compares closely with the isotopic stasis recorded in the Society Cliffs Formation and supports broad lithologic correlation.

The Narssârssuk Formation, Thule Group, consists of $>670 \mathrm{~m}$ of peritidal siliciclastic and carbonate rocks preserved in the Wolstenholme Fjord region of northwest Greenland (Kurtz and Wales 1951; Jackson 1986; Dawes 1997). Recent reevaluation of facies characteristics between Baffin Island and Greenland (Hofmann and Jackson 1996) suggests that transitions in the Narssârssuk formation from redbeds and gypsiferous dolostone, through open-marine limestone and dolostone, to reddish sandstone are strikingly similar to depositional trends of the Society Cliffs, Victor Bay, and Athole Point formations. Only 12 analyses are available from the Narssârssuk Formation (Table 5) (A.J. Kaufman and J.M. Hayes, unpublished data). These analyses are of limited stratigraphic utility in that $(i)$ there is little diagenetic control; and (ii) samples are assigned only to relative position within geologic members, and not to measured stratigraphic positions. A confident interpretation cannot be ensured without more precise stratigraphic information; however, carbon isotopic values broadly support deposition coeval with Bylot Supergroup strata. In contrast to the Hunting Formation, general trends within the Narssârssuk Formation show a strong similarity to the entire carbonate sequence preserved on Baffin Island: values near $2 \%$ followed by a drop in values to near $0 \%$ and a subsequent rise back to more positive values (to $2.7 \%$ ). On Baffin Island, af- ter deposition of the Society Cliffs Formation (2-4\%o), near $0 \%$ values occur near the base of the Victor Bay Formation. The isotopic profile on Baffin Island does not return to values $>1.3 \%$ until the uppermost Victor Bay Formation, with values exceeding $2 \%$ exclusively within Athole Point Formation strata (Fig. 6). In light of these carbon isotopic trends, the uppermost Narssârssuk Formation appears coeval with the Athole Point Formation, whereas the lower and middle Narssârssuk Formation is more closely correlatable to the Society Cliffs and Victor Bay formations. The occurrence in the Narssârssuk Formation of isotopic signatures similar to Athole Point strata is particularly interesting, as the Athole Point Formation is reported absent between the central Borden Basin and Greenland (Jackson and Iannelli 1981). Further documentation of fine-scale stratigraphic and chemostratigraphic relationships, particularly of sedimentary successions on Bylot Island and northwest Greenland, should provide the data necessary to track lithologic units between these geographically disparate sections and to determine depositional trends during basin evolution.

Whereas the record of carbon isotopic variation broadly supports coeval deposition for strata from Baffin Island, Somerset Island, and northwest Greenland, carbon isotopic values recorded in the aforementioned strata are significantly different from those observed in the Shaler Supergroup of northwestern Canada. Sedimentary rocks from the Shaler Supergroup preserve a distinctive carbon isotopic profile: basal values of $\sim 0 \%$, an interval between $3 \%$ and $4 \%$, and a peak near $6 \%$ followed by a significant negative excursion (Kaufman and Knoll 1995). This pattern of isotopic variation can be correlated globally and suggests deposition during the interval between $\sim 730$ and $850 \mathrm{Ma}$ (Kaufman and Knoll 1995). Deposition during this interval is consistent with a maximum age of $1077 \pm 3$ Ma for the Shaler Supergroup determined by detrital zircon analyses (Rainbird et al. 1992), yet inconsistent with paleomagnetic evidence from the Bylot Supergroup (Fahrig et al. 1981). Isotopic data presented here therefore support the hypothesis that deposition of thick sedimentary packages in eastern Laurentia oc- 
Fig. 8. Mesoproterozoic to Neoproterozoic chemostratigraphic curve. The thin black line represents the well-constrained carbon isotopic curve for the later Neoproterozoic (modified from Kaufman and Knoll 1995). The thicker grey line represents a preliminary compilation of Mesoproterozoic to earliest Neoproterozoic carbon isotopic compositions, including the present study. Labelled bars represent the approximate chronology of Mesoproterozoic successions for which both isotopic and age data are available (see text for age references). AL, Allamoore Formation; BT, Belt Supergroup; BY, Bylot Supergroup (this study); CL, Callana Group; GV, Grenville Supergroup; MS, Mescal Formation; SH, Shaler Supergroup; TK, Turukhansk Group.

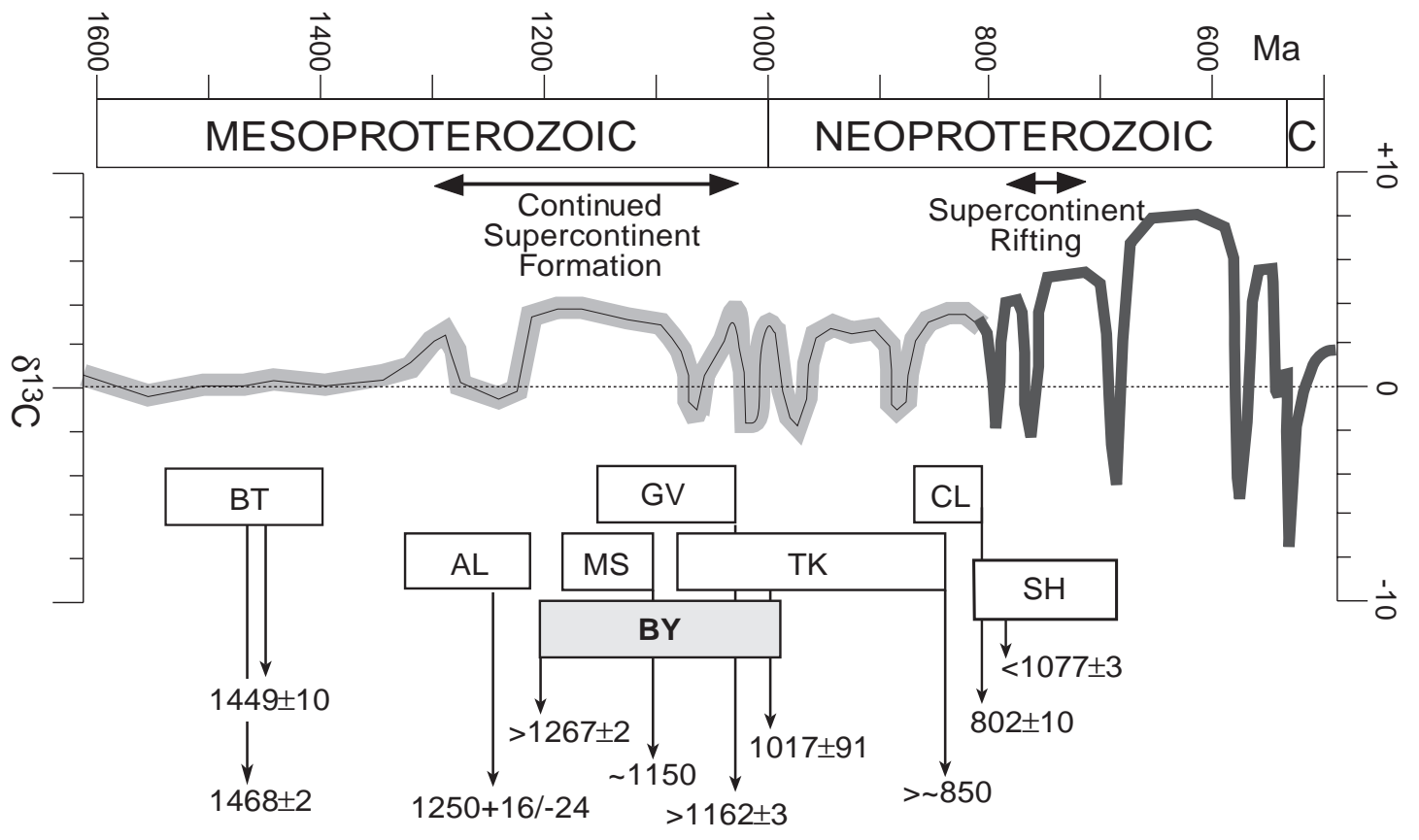

curred prior to deposition of the Shaler Supergroup and record an interval represented in northwestern Laurentia by a major unconformity.

\section{Extending the global carbon isotopic record}

A review of published literature reveals that several Mesoproterozoic successions do record a restricted range of $\delta^{13} \mathrm{C}$, typically near $0.0 \pm 1.0 \%$. These include the Bangemall Group, Australia (Buick et al. 1995); Billyakh Group, Siberia (Knoll et al. 1995); Carswell Formation, Canada (Abell et al. 1989); Changcheng, Jixian, and Luonan groups, China (Xiao et al. 1997); and Belt Supergroup, Montana (Frank et al. 1997). Where available, age constraints for these units suggest deposition prior to $\sim 1300 \mathrm{Ma}$ (age constraints are from the following sources: Bangemall Formation (Gee et al. 1976; D.R. Nelson, quoted in Buick et al. 1995); Billyakh Group (Knoll et al. 1995); Carswell Formation (Bell 1981; Ramaekers 1981; Clauer et al. 1985); Changcheng, Jixian, and Luonan groups (cited in Xiao et al. 1997); Belt Supergroup (Zartman et al. 1982; Aleinikoff et al. 1996; Anderson and Davis 1995, and references therein)). In contrast, numerous other Mesoproterozoic to early Neoproterozoic successions are characterized by more positive carbon isotopic values, with $\delta^{13} \mathrm{C}$ typically $3.5 \pm 0.5 \%$ o. Successions showing elevated $\delta^{13} \mathrm{C}$ values include the Bylot Supergroup and Hunting Formation, Canada; the Narssârssuk Formation, Greenland; the Allamoore Formation, Texas (L.C. Kah and J.K. Bartley, unpublished data); the Grenville Supergroup, New York (Whelan et al. 1990); the Mescal
Limestone, Arizona (Beeunas and Knauth 1985); the Turukhansk Group, Siberia (Knoll et al. 1995); the Raipur (Schidlowski et al. 1975; Murti and Aswathanarayana 1982), Kurnool (Schidlowski et al. 1975; Dasari 1989), and Badami and Bhima (Sathyanarayan et al. 1987) groups, India; and the Neoproterozoic Atar Group, Mauritania (Fairchild et al. 1990) and Callana Dolomite, Australia (Lambert 1984). Of these units, the Bylot Supergroup (Heaman et al. 1990; LeCheminant and Heaman 1991), Hunting Formation (Jones and Fahrig 1978), Narssârssuk Formation (Dawes 1997), Allamoore Formation (Roths 1993), Grenville Supergroup (Heaman et al. 1986; van Breemen and Davidson 1988), Mescal Limestone (Livingston and Damon 1968; Silver 1978; Heaman and Grotzinger 1992), Turukhansk Group (Ovchinnikova et al. 1995), and Callana Dolomite (Fanning et al. 1986) contain evidence supporting deposition between $\sim 1300$ and $\sim 800 \mathrm{Ma}$. Combined, these data suggest that the latest Mesoproterozoic to earliest Neoproterozoic may represent an isotopically recognizable interval in Earth history.

Compilation of published age and isotopic data therefore allows construction of a preliminary global isotopic curve which suggests that the Mesoproterozoic and Neoproterozoic eras can be divided into three distinct isotopic intervals: (i) isotopically invariant $0 \%$, (ii) subdued variation between approximately $-1 \%$ and $\sim 3.5 \%$, and (iii) wide variation with a significantly elevated baseline $>4-5 \%$ (Fig. 8). Although the timing of isotopic transitions is poorly constrained, moderately positive values recorded in the Allamoore Formation and Bylot Supergroup indicate that the observed carbon isotopic shift must have occurred no later than $\sim 1270-1250$ Ma. Furthermore, age data from the 
Mesoproterozoic to Neoproterozoic Turukhansk Group and the Neoproterozoic Shaler Supergroup and Callana Group help to define the transition between the subdued variation of the Mesoproterozoic to Neoproterozoic transition and the large isotopic excursions characteristic of the later Neoproterozoic (Kaufman and Knoll 1995). Turukhansk Group strata show an isotopic profile dominated by background values near 3\%o (Knoll et al. 1995). Basal formations are constrained to be older than $1017 \pm 91(\mathrm{~Pb}-\mathrm{Pb}$ carbonate; Ovchinnikova et al. 1995) and show excursions similar to those observed in upper Bylot Supergroup strata. Upper Turukhansk Group strata reveal a second stable $\sim 3 \%$ plateau and contain a variety of acanthomorphic acritarchs indicating a Neoproterozoic age, though older than regional $850 \mathrm{Ma}$ thermal overprinting (Knoll et al. 1995). Callana carbonates, constrained in age to be older than the Rook tuff (802 \pm 10 Ma, U-Pb zircon; Fanning et al. 1986), reveal carbon isotopic values of $\sim 3 \%$ (Lambert 1984), suggesting a continuation of moderately elevated isotopic values well into the Neoproterozoic. If Callana carbonates can be shown to be correlative with the Mikkelson Island Formation, lower Shaler Supergroup, as proposed by Rainbird et al. (1996), this age may also mark the time after which carbon isotopes rise dramatically to typically high Neoproterozoic values (Kaufman and Knoll 1995).

\section{Conclusions}

Isotopic values from the Bylot Supergroup and genetically related successions on Somerset Island and in northwest Greenland add to a growing base of stratigraphically and diagenetically well-constrained data that allow us to determine the primary carbon isotopic compositions of ancient oceans. Data reveal distinct stratigraphic trends in $\delta^{13} \mathrm{C}$, with intervals of moderate enrichment (approx. +3.5\%o) punctuated by excursions to slightly negative values (approx. $-1 \%$ ), indicating that even relatively subdued patterns of isotopic variation may be suitable for testing regional stratigraphic correlations. Moreover, the stratigraphic continuity of Bylot Supergroup data provides data essential to the development of a global isotopic curve for the Mesoproterozoic.

Carbon isotopic evidence suggests that the Mesoproterozoic and Neoproterozoic eras can be divided into three distinct isotopic intervals. An extended interval of isotopic quiescence $\left(\delta^{13} \mathrm{C}=0.0 \pm 1.0 \%\right.$ o $)$ occurred from $\sim 1600$ to $1300 \mathrm{Ma}$, suggesting a dynamic stability of the Earth's oxidized and reduced reservoirs (Buick et al. 1995; Brasier and Lindsay 1998). Beginning $1300 \mathrm{Ma}$, a new baseline was established with moderately positive isotopic ratios $\left(\delta^{13} \mathrm{C}=3.5 \pm 1.0 \%\right.$ ), punctuated by several brief, negative excursions; these elevated isotopic ratios prevailed through the end of the Mesoproterozoic and well into the Neoproterozoic. Finally, between $\sim 800$ and $\sim 780 \mathrm{Ma}$ (the age of intrusive dykes in the lowermost Mackenzie Mountains, an equivalent to the lower Shaler Supergroup; Jefferson and Parrish 1989) carbon isotopes begin to record the extreme positive and negative values that characterize later Neoproterozoic successions (cf. Kaufman and Knoll 1995).

That first-order transitions in carbon isotopic ratios appear to coincide with the initial assembly $(\sim 1300 \mathrm{Ma})$ and subsequent breakup $(\sim 800 \mathrm{Ma})$ of the Rodinian supercontinent may indicate a biogeochemical linkage between tectonic events and ocean chemistry. Onset of elevated carbon isotopic values coeval with orogenic events suggests increased organic carbon burial with either tectonically driven sedimentation or development of restricted basins (Knoll et al. 1986; Derry et al. 1992). Increased burial in turn shields organic matter from oxidation and may have resulted in a net increase in the oxidation state of the oceans and atmosphere (Des Marais et al. 1992) and subsequent biological innovation (Canfield and Teske 1996). Increased resolution of the Earth's carbon isotopic record and correlation of this record with independent sedimentologic, tectonic, and biogeochemical records may therefore lead to a greater understanding of the Earth's evolving biosphere.

\section{Acknowledgments}

Field and laboratory work was supported in part by the National Geographic Society (Kah and Knoll), National Aeronautics and Space Administration (Knoll), National Sciences and Engineering Research Council of Canada (Narbonne), and the National Science Foundation (Kaufman). We thank J. Sullivan and J. Harrington for field assistance; R. Rainbird, N. Butterfield, and K. Swett for generous contribution of stratigraphic information and samples from the Hunting Formation; J. Hayes for contributing isotopic data from the Narssârssuk Formation; and M. Emmons, B. McDonough, R. Pflaum, and S. Jacobsen for assistance in elemental and isotopic analysis. We thank also J. Bartley, G. Ross, and M. Savard for thoughtful criticism of the manuscript.

\section{References}

Abell, P.I., McClory, J., Hendry, H.E., and Wheatley, K.L. 1989. Stratigraphic variations in carbon and oxygen isotopes in the dolostone of the Carswell Formation (Proterozoic) of northern Saskatchewan. Canadian Journal of Earth Sciences, 26: 23182326.

Aleinikoff, J.N., Evans, K.V., Fanning, C.M., Obradovich, J.D., Ruppel, E.T., Zeig, J.A., and Steinmetz, J.C. 1996. SHRIMP U-Pb ages of felsic igneous rocks, Belt Supergroup, western Montana. Geological Society of America, Abstracts with Programs, 28: A376.

Anderson, H.E., and Davis, D.W. 1995. U-Pb geochronology of the Moyie sills, Purcell Supergroup, southeastern British Columbia: implications for the Mesoproterozoic geological history of the Purcell (Belt) basin. Canadian Journal of Earth Sciences, 32: 1180-1193.

Banner, J.L., and Hanson, G.N. 1990. Calculation of simultaneous isotopic and trace element variations during water-rock interaction with applications to carbonate diagenesis. Geochimica et Cosmochimica Acta, 54: 3123-3137.

Bartley, J.K., Pope, M., Knoll, A.H., Semikhatov, M.A., and Petrov, P.Yu. 1998. A Vendian-Cambrian boundary succession from the northwestern margin of the Siberian platform: stratigraphy, paleontology, chemostratigraphy, and correlation. Geological Magazine, 135: 473-494.

Beeunas, M.A., and Knauth, L.P. 1985. Preserved stable isotopic signature of subaerial diagenesis in the 1.2-b.y. Mescal Limestone, central Arizona: implications for the timing and development of a terrestrial plant cover. Geological Society of America Bulletin, 96: 737-745. 
Bell, K. 1981. A review of the geochronology of the Precambrian in Saskatchewan - some clues to uranium mineralization. Mineralogical Magazine, 44: 371-378.

Bond, C.G., Nickeson, P.A., and Kominz, M.A. 1984. Breakup of a supercontinent between 623 and $555 \mathrm{Ma}$; new evidence and implications for continental histories. Earth and Planetary Science Letters, 70: $325-345$.

Bralower, T.J., Thomas, D.J., Zachos, J.C., Hirschmann, M.M., Röhl, U., Sigurdsson, H., Thomas, E., and Whitney, D.L. 1997. High-resolution records of the late Paleocene thermal maximum and circum-Caribbean volcanism: is there a causal link? Geology, 25: 963-966.

Brasier, M.D., and Lindsay, J.F. 1998. A billion years of environmental stability and the emergence of eukaryotes; new data from northern Australia. Geology, 26: 555-558.

Brasier, M.D., Shields, G., Kuleshov, V.N., and Zhegallo, L.A. 1996. Integrated chemo- and biostratigraphic calibration of early animal evolution: Neoproterozoic - Early Cambrian of southwest Mongolia. Geological Magazine, 133: 445-485.

Broecker, W.S., and Peng, T.S. 1982. Tracers in the sea. Eldigio Press, New York.

Buick, R., Des Marais, D.J., and Knoll, A.H. 1995. Stable isotopic compositions of carbonates from the Mesoproterozoic Bangemall Group, northwestern Australia. Chemical Geology, 123: $153-171$.

Burdett, J.W., Grotzinger, J.P., and Arthur, M.A. 1990. Did major changes in the stable isotopic composition of Proterozoic seawater occur? Geology, 18: 227-230.

Canfield, D.E., and Teske, A. 1996. Late Proterozoic rise in atmospheric oxygen concentration inferred from phylogenetic and sulphur-isotope studies. Nature (London), 382: 127-132.

Carpenter, S.J., Lohmann, K.C., Holden, P., Walter, L.M., Huston, T.J., and Halliday, A.N. $1991 . \delta^{18} \mathrm{O}$ values, $87 \mathrm{Sr} / 86 \mathrm{Sr}$ and $\mathrm{Sr} / \mathrm{Mg}$ ratios of Late Devonian marine calcite. Implications for the composition of ancient seawater. Geochimica et Cosmochimica Acta, 55: 1991-2010.

Christie, K.W., and Fahrig, W.F. 1983. Paleomagnetism of Borden dykes of Baffin Island and its bearing on the Grenville Loop. Canadian Journal of Earth Sciences, 20: 275-289.

Clauer, N., Ey, F., and Gauthier-LaFaye, F. 1985. K-Ar dating of different rock types from Cluff Lake uranium ore deposits (Saskatchewan, Canada). In The Carswell structure uranium deposits, Saskatchewan. Edited by R. Lainé, D. Alonso, and M. Svab. Geological Association of Canada, Special Paper 29, pp. 47-53.

Clayton, R.H., and Thorpe, L. 1982. Geology of the Nanisivik zinc-lead deposit. In Precambrian sulphide deposits. Edited by R.W. Hutchinson, C.D. Spence, and J.M. Franklin. Geological Association of Canada, Special Paper 25, pp. 739-758.

Coleman, M.L. 1993. Microbial processes: controls on the shape and composition of carbonate concretions. Marine Geology, 113: $127-140$.

Dalziel, I.W.D. 1991. Pacific margins of Laurentia and East Antarctica - Australia as a conjugate rift pair: evidence and implications for an Eocambrian supercontinent. Geology, 19: 598601.

Dasari, M.R. 1989. Isotopic variations in limestones from Kurnool sediments in Caddapah Basin, India. Journal of the Geological Society of India, 35: 447-449.

Dawes, P.R. 1997. The Proterozoic Thule Group, Greenland and Canada: history, lithostratigraphy, and development. Geology of Greenland Survey, Bulletin 174.

Derry, L.A., Kaufman, A.J., and Jacobsen, S.B. 1992. Sedimentary cycling and environmental change in the late Proterozoic: evi- dence from stable and radiogenic isotopes. Geochimica et Cosmochimica Acta, 56: 1317-1329.

Des Marais, D.J., Strauss, H., Summons, R.E., and Hayes, J.M. 1992. Carbon isotopic evidence for the step-wise oxidation of the Proterozoic environment. Nature (London), 359: 605-609.

Dickens, G.R., O'Neil, J.R., Rea, D.K., and Owen, R.M. 1995. Dissociation of oceanic methane hydrate as a cause of the carbon isotope excursion at the end of the Paleocene. Paleoceanography, 10: 965-971.

Dixon, J. 1974. Revised stratigraphy of the Hunting Formation (Proterozoic), Somerset Island, Northwest Territories. Canadian Journal of Earth Sciences, 11: 635-642.

Fahrig, W.F., Irving, E., and Jackson, G.D. 1971. Paleomagnetism of the Franklin diabases. Canadian Journal of Earth Sciences, 8: 455-467.

Fahrig, W.F., Christie, K.W., and Jones, D.L. 1981. Paleomagnetism of the Bylot Basins: evidence for Mackenzie continental tensional tectonics. In Proterozoic Basins of Canada. Edited by F.H.A. Campbell. Geological Survey of Canada, Paper 81-10, pp. 303-312.

Fairchild, I.J., and Spiro, B. 1987. Petrological and isotopic implications of some contrasting Late Precambrian carbonates, NE Spitsbergen. Sedimentology, 34: 973-989.

Fairchild, I.J., Marshall, J.D., and Bertrand-Sarfati, J. 1990. Stratigraphic shifts in carbon isotopes from Proterozoic stromatolitic carbonates (Mauritania); influences of primary mineralogy and diagenesis. American Journal of Science, 290A: 46-79.

Fanning, C.M., Ludwig, K.R., Forbes, B.G., and Preiss, W.V. 1986. Single and multiple grain $\mathrm{U}-\mathrm{Pb}$ zircon analyses for the early Adeladian Rook Tuff, Willouran Ranges, South Australia. Abstracts of the Geological Society of Australia, 15: 71-72.

Frank, T.D., Lyons, T.W., and Lohmann, K.C. 1997. Isotopic evidence for the paleoenvironmental evolution of the Mesoproterozoic Helena Formation, Belt Supergroup, Montana. Geochimica et Cosmochimica Acta, 61: 5023-5041.

Galley, A.G., Jackson, G.D., and Iannelli, T.R. 1983. Neohelikian subaerial basalts with ocean-floor type chemistry, northwestern Baffin Island. Geological Association of Canada - Mineralogical Association of Canada, Program with Abstracts, 8: A25.

Gee, R.D., De Laeter, J.R., and Drake, J.R. 1976. The geology and geochronology of altered rhyolite from the lower part of the Bangemall Group near Tangadee, Western Australia. Annual Report of the Geological Survey of Western Australia, 1975: 112-117.

Geldsetzer, H. 1973. The tectono-sedimentary development of an algal-dominated Helikian succession on northern Baffin Island, N.W.T. Geological Association of Canada Memoir, 19: 99-126.

Ghazban, F., Schwarcz, H.P., and Ford, D.C. 1992. Multistage dolomitization in the Society Cliffs Formation, northern Baffin Island, Northwest Territories, Canada. Canadian Journal of Earth Sciences, 29: 1459-1473.

Grotzinger, J.P., and Knoll, A.H. 1995. Anomalous carbonate precipitates: is the Precambrian the key to the Permian? Palaios, 10: $578-596$.

Hayes, J.M., Popp, B.N., Takigiku, R., and Johnson, M.W. 1989. An isotopic study of biogeochemical relationships between carbonates and organic carbon in the greenhorn formation. Geochimica et Cosmochimica Acta, 53: 2961-2972.

Heaman, L.M., and Grotzinger, J.P. 1992. 1.08 Ga diabase sills in the Pahrump Group, California: implications for development of the Cordilleran miogeocline. Geology, 20: 637-640.

Heaman, L.M., McNutt, R.H., and Krogh, T.E. 1986. Geological Significance of $\mathrm{U}-\mathrm{Pb}$ and $\mathrm{Rb}-\mathrm{Sr}$ ages for two pre-tectonic gran- 
ites from the CMB, Ontario. In the Grenville Province. Edited by J.M. Moore, A. Davidson, and A.J. Baer. Geological Association of Canada, Special Paper 31, pp. 209-222.

Heaman, L.M., LeCheminant, A.N., and Rainbird, R.H. 1990. A $\mathrm{U}-\mathrm{Pb}$ baddeleyite study of Franklin igneous events. Geological Association of Canada, Programs with Abstracts, 15: A55.

Hemming, N.G., Meyers, W.J., and Grams, J.C. 1989. Cathodoluminescence in diagenetic calcites: the roles of $\mathrm{Fe}$ and $\mathrm{Mn}$ as deduced from electron probe and spectrophotometric measurements. Journal of Sedimentary Petrology, 59: 404-411.

Hoffman, P.F. 1991. Did the breakout of Laurentia turn Gondwanaland inside-out? Science (Washington, D.C.), 252: 1409-1412.

Hofmann, H.J., and Jackson, G.D. 1991. Shelf-facies microfossils from the Uluksan Group (Proterozoic Bylot Supergroup), Baffin Island, Canada. Journal of Paleontology, 65: 361-382.

Hofmann, H.J., and Jackson, G.D. 1994. Shale-facies microfossils from the Proterozoic Bylot Supergroup, Baffin Island, Canada. Paleontological Society Memoir, 37: 1-39.

Hofmann, H.J., and Jackson, G.D. 1996. Notes on the geology and paleontology of the Proterozoic Thule Group, Ellesmere Island, Canada, and North-west Greenland. Geological Survey of Canada, Bulletin 495, pp. 1-26.

Hoffman, P.F., Kaufman, A.J., Halverson, G.P., and Schrag, D.P. 1998. A Neoproterozoic snowball Earth. Science (Washington, D.C.), 281: 1342-1346.

Iannelli, T.R. 1979. Stratigraphy and depositional history of some Upper Proterozoic sedimentary rocks on Northwestern Baffin Island, District of Franklin. In Current research, part A. Geological Survey of Canada, Paper 79-1A, pp. 45-56.

Jackson, G.D. 1974. Interpretation of whole-rock K-Ar ages for some related samples from west of Arctic Bay. In Age determinations and geological studies, $\mathrm{K}-\mathrm{Ar}$ isotropic studies, Report XII. Edited by R.K. Wanless, R.D. Stevens, G.R. Lachance, and R.N.D. Delabio. Geological Survey of Canada, Paper 74-2, pp. 24-25.

Jackson, G.D. 1986. Notes on the Proterozoic Thule Group, northern Baffin Bay. In Current research, part A. Geological Survey of Canada, Paper 86-1A, pp. 541-552.

Jackson, G.D., and Davidson, A. 1978. Bylot Island map-area, District of Franklin. Geological Survey of Canada, Paper 74-29, pp. 1-7.

Jackson, G.D., and Iannelli, T.R. 1981. Rift-related cyclic sedimentation in the Neohelikian Borden Basin, northern Baffin Island. In Proterozoic basins of Canada. Edited by F.H.A. Campbell. Geological Survey of Canada, Paper 81-10, pp. 269-302.

Jackson, G.D., Davidson, A., and Morgan, W.C. 1975. Geology of the Pond Inlet map-area, Baffin Island, District of Franklin. Geological Survey of Canada, Paper 74-25, pp. 1-33.

Jackson, G.D., Iannelli, T.R., Narbonne, G.M., and Wallace, P.J. 1978. Upper Proterozoic sedimentary and volcanic rocks of northwestern Baffin Island. Geological Survey of Canada, Paper 78-14, pp. 1-15.

Jackson, G.D., Iannelli, T.R., Knight, R.D., and Lebel, D. 1985. Neohelikian Bylot Supergroup of Borden Rift Basin, northwestern Baffin Island, District of Franklin. In Current research, part A. Geological Survey of Canada, Paper 85-1A, pp. 639-649.

Jackson, G.D., Hunt, P.A., Loveridge, W.D., and Parrish, R.R. 1990. Reconnaissance geochronology of Baffin Island, N.W.T. In Radiogenic age and isotopic studies, Report 3. Geological Survey of Canada, Paper 89-2, pp. 123-148.

James, N.P., Narbonne, G.M., and Sherman, A.G. 1998. Molartooth carbonates: shallow subtidal facies of the mid- to late Proterozoic. Journal of Sedimentary Research, 68: 716-722.
Jefferson, C.W., and Parrish, R.R. 1989. Late Proterozoic stratigraphy, U-Pb zircon ages, and rift tectonics, Mackenzie Mountains, northwestern Canada. Canadian Journal of Earth Sciences, 26: 1784-1801.

Jones, D.L., and Fahrig, W.F. 1978. Paleomagnetism and age of the Aston dykes and Savage Point sills of the Boothia Uplift, Canada. Canadian Journal of Earth Sciences, 15: 1605-1612.

Kah, L.C. 1997. Sedimentological, geochemical, and paleobiological interactions on a Mesoproterozoic carbonate platform: Society Cliffs Formation, northern Baffin Island, Arctic Canada. Ph.D. dissertation, Harvard University, Cambridge, Mass.

Kah, L.C., and Knoll, A.H. 1996. Microbenthic distribution in Proterozoic tidal flats: environmental and taphonomic considerations. Geology, 24: 79-81.

Kaufman, A.J., and Knoll, A.H. 1995. Neoproterozoic variations in the carbon isotopic composition of seawater: stratigraphic and biogeochemical implications. Precambrian Research, 73: 27-49.

Kaufman, A.J., Hayes, J.M., Knoll, A.H., and Germs, G.J.B. 1991. Isotopic compositions of carbonates and organic carbon from upper Proterozoic successions in Namibia: stratigraphic variation and the effects of diagenesis and metamorphism. Precambrian Research, 49: 301-327.

Kaufman, A.J., Knoll, A.H., and Narbonne, G.M. 1997. Isotopes, ice ages, and terminal Proterozoic earth history. Proceedings of the National Academy of Sciences, 94: 6600-6605.

Knight, R.D., and Jackson, G.D. 1994. Sedimentology and stratigraphy of the Mesoproterozoic Elwin Subgroup (Aqigilik and Sinasiuvik formations), uppermost Bylot Supergroup, Borden Rift Basin, northern Baffin Island. Geological Survey of Canada, Bulletin 455 .

Knoll, A.H. 1991. End of the Proterozoic eon. Scientific American, 265: $64-73$.

Knoll, A.H., and Walter, M. 1992. Latest Proterozoic stratigraphy and Earth history. Nature (London), 356: 673-678.

Knoll, A.H., Hayes, J.M., Kaufman, A.J., Swett, K., and Lambert, I.B. 1986. Secular variation in carbon isotope ratios from Upper Proterozoic successions of Svalbard and East Greenland. Nature (London), 321: 832-838.

Knoll, A.H., Kaufman, A.J., and Semikhatov, M.A. 1995. The carbon isotopic composition of Proterozoic carbonates: Riphean successions from northwestern Siberia (Anabar Massif, Turukhansk Uplift). American Journal of Science, 295: 823850.

Knoll, A.K., Bamback, R.K., Canfield, D.E., and Grotzinger, J.P. 1996. Comparative Earth history and the late Permian mass extinction. Science (Washington, D.C.), 273: 452-457.

Krogh, T.E., Corfu, F., Davis, D.W., Dunning, G.R., Heaman, L.M., Kamo, S.L., and Machado, N. 1987. Precise U-Pb isotopic ages of diabase dykes and mafic to ultramafic rocks using trace amounts of baddeleyite and zircon. In Mafic dyke swarms. Edited by H.C. Halls and W.F. Fahrig. Geological Association of Canada, Special Paper 34, pp. 147-152.

Kurtz, V.E., and Wales, D.B. 1951. Geology of the Thule area, Greenland. Oklahoma Academy of Sciences, 1950: 83-89.

Lambert, I.B. 1984. Genesis of late Proterozoic copper mineralization, Copper Claim, South Australia. Economic Geology and the Bulletin of the Society of Economic Geologists, 79: 461-475.

Land, L.S. 1980. The isotopic and trace element geochemistry of dolomite: the state of the art. In Concepts and models of dolomitization. Edited by D.H. Zenger, J.B. Dunham, and R.L. Ethington. Society of Economic Paleontologists and Mineralogists, Special Publication 28, pp. 87-110. 
LeCheminant, A.N., and Heaman, L.M. 1989. Mackenzie igneous events, Canada: Middle Proterozoic hotspot magmatism associated with ocean opening. Earth and Planetary Science Letters, 96: $38-48$.

LeCheminant, A.N., and Heaman, L.M. 1991. U-Pb ages for the 1.27 Ga Mackenzie igneous events, Canada: support for a plume initiation model. Geological Association of Canada. Programs with Abstracts, 16: A-73.

Livingston, D.E., and Damon, P.E. 1968. The age of stratified rock sequences in central Arizona and northern Sonora. Canadian Journal of Earth Sciences, 5: 763-772.

Marshall, J.D. 1992. Climatic and oceanographic signals from the carbonate rock record and their preservation. Geological Magazine, 129: 143-160.

Murti, K.S., and Aswathanarayana, U. 1982. Chemical and isotopic indicators of paleoenvironments of the late Proterozoic limestones of the Chhattisgarh Basin, India. In Abstracts of the International Conference on Geochronology, Cosmochronology, and Isotope Geology, Vol. 5, p. 258.

Narbonne, G.M., and James, N.P. 1996. Mesoproterozoic deepwater reefs from Borden Peninsula, Arctic Canada. Sedimentology, 43: 827-848.

Narbonne, G.M., Kaufman, A.J., and Knoll, A.H. 1994. Integrated chemostratigraphy and biostratigraphy of the upper Windermere Supergroup, northwestern Canada: implications for Neoproterozoic correlations and the early evolution of animals. Geological Society of America Bulletin, 106: 1281-1292.

Olson, R.A. 1984. Genesis of paleokarst and stratabound $\mathrm{Zn}-\mathrm{Pb}$ sulfide deposits in a Proterozoic dolostone, northern Baffin Island, Canada. Economic Geology, 79: 1056-1103.

Ovchinnikova, G.V, Semikhatov, M.A., Gorokhov, I.M., Belyatskii, B.V., Vasilieva, I.M., and Levskii, L.K. 1995. U-Pb systematics of Pre-Cambrian carbonates: the Riphean SukhayaTunguska Formation in the Turukhansh Uplift, Siberia. Lithology and Mineral Resources (English Translation), 30: 477-487.

Pehrsson, S.J., and Buchan, K.L. 1993. Borden dykes interpreted as remagnetized Franklin dykes based on $\mathrm{U}-\mathrm{Pb}$ baddeleyite geochronology and paleomagnetic re-interpretation. Geological Association of Canada, Program with Abstracts, 19: A87.

Pelechaty, S.M., Kaufman, A.J., and Grotzinger, J.P. 1996. Evaluation of $\delta^{13} \mathrm{C}$ isotope stratigraphy for intrabasinal correlation: Vendian strata of northeast Siberia. Geological Society of America Bulletin, 108: 992-1003.

Pierson, B J. 1981. The control of cathodoluminescence in dolomite by iron and manganese. Sedimentology, 28: 601-610.

Rainbird, R.H., Heaman, L.M., and Young, G.M. 1992. Sampling Laurentia: detrital zircon geochronology offers evidence for an extensive Neoproterozoic river system originating from the Grenville orogen. Geology, 20: 351-354.

Rainbird, R.H., Jefferson, C.W., Hildebrand, R.S., and Worth, J.K. 1994. The Shaler Supergroup and revision of Neoproterozoic stratigraphy in Amundsen Basin, Northwest Territories. In Current research, part C. Geological Survey of Canada, Paper 1994-C, pp. 61-70.

Rainbird, R.H., Jefferson, C.W., and Young, G.M. 1996. The early Neoproterozoic sedimentary Succession B of northwestern Laurentia: correlations and paleogeographic significance. Geological Society of America Bulletin, 108: 454-470.

Ramaekers, P. 1981. Hudsonian and Helikian basins of the Athabasca region, northern Saskatchewan. In Proterozoic basins of Canada. Edited by F.H.A. Campbell. Geological Survey of Canada, Paper 81-10, pp. 219-233.

Reinson, G.E., Kerr, J.W., and Stewart, W.D. 1976. Stratigraphic field studies, Somerset Island, District of Franklin. In Report of activities, part A. Geological Survey of Canada, Paper 76-1A, pp. 497-499.

Roths, P.J. 1993. Geochemical and geochronological studies of the Grenville-age (1250-1000 Ma) Allamoore and Hazel formations, Hudspeth and Culberson counties, West Texas. Geological Society of America, Field Trip Guidebook, pp. 11-35.

Rush, P.F., and Chafetz, H.S. 1990. Fabric retentive, non-luminescent brachiopods as indicators of original $\delta^{13} \mathrm{C}$ and $\delta^{18} \mathrm{O}$ compositions: a test. Journal of Sedimentary Petrology, 60: 968-981.

Saltzman, M.R., Davidson, J.P., Holden, P., Runnegar, B., and Lohmann, K.C. 1995. Sea level-driven changes in ocean chemistry at an Upper Cambrian extinction horizon. Geology, 23: 893896.

Sathyanarayan, S., Arneth, J.D., and Schidlowski, M. 1987. Stable isotope geochemistry of sedimentary carbonates from the Proterozoic Kaladagi, Badami and Bhima groups, Karnataka, India. Precambrian Research, 37: 147-156.

Savard, M.M., Veizer, J., and Hinton, R. 1995. Cathodoluminescence at low $\mathrm{Fe}$ and Mn concentrations: a SIMS study of zones in natural calcite. Journal of Sedimentary Research, A65: 208-213.

Schidlowski, M., Eichmann, R., and Junge, C.E. 1975. Precambrian sedimentary carbonates: carbon and oxygen isotope geochemistry and implications for the terrestrial oxygen budget. Precambrian Research, 2: 1-69.

Schidlowski, M., Hayes, J.M., and Kaplan, I.R. 1983. Isotopic inferences of ancient biochemistries: carbon, sulfur, hydrogen, and nitrogen. In Earth's earliest biosphere. Edited by J.W. Schopf. Princeton University Press, Princeton, N.J., pp. 149-187.

Sheppard, S.M.F., and Schwarcz, H.P. 1970. Fractionation of carbon and oxygen isotopes and magnesium between coexisting metamorphic calcite and dolomite. Contributions to Mineralogy and Petrology, 26: 161-198.

Silver, L.T. 1978. Precambrian formations and Precambrian history in Cochise County, southeastern Arizona. New Mexico Geological Society Field Conference Guidebook, 29: 157-163.

Tuke, M.F., Dineley, D.L., and Rust, B.R. 1966. The basal sedimentary rocks in Somerset Island, N.W.T. Canadian Journal of Earth Sciences, 3: 697-711.

van Breemen, O., and Davidson, A. 1988. U-Pb zircon ages of granites and syenites in the Central Metasedimentary Belt, Grenville Province, Ontario. In Radiogenic age and isotopic studies, Report 2. Edited by J.K. Mortensen. Geological Survey of Canada, Paper 88-2, pp. 45-50.

Veizer, J. 1983. Trace elements and isotopes in sedimentary carbonates. Carbonates; mineralogy and chemistry. Edited by R.J. Reeder. Reviews in Mineralogy, 11: 265-300.

Veizer, J., and Hoefs, J. 1976. The nature of $180 / 160$ and $13 \mathrm{C} / 12 \mathrm{C}$ secular trends in sedimentary carbonate rocks. Geochimica et Cosmochimica Acta, 40: 1387-1395.

Veizer, J., Plumb, K.A., Clayton, R.N., Hinton, R.W., and Grotzinger, J.P. 1992. Geochemistry of Precambrian carbonates: V. Late Paleoproterozoic seawater. Geochimica et Cosmochimica Acta, 56: 2487-2501.

Whelan, J.F., Rye, R.O., deLorraine, W., and Ohmoto, H. 1990. Isotopic geochemistry of a mid-Proterozoic evaporite basin: Balmat, New York. American Journal of Science, 290: 396-424.

Wickham, S.M., and Peters. M.T. 1993. High $\delta^{13}$ C Neoproterozoic carbonate rocks in western North America. Geology, 21: 165168.

Xiao, S., Knoll, A.H., Kaufman, A.J., Yin, L., and Yun, Z. 1997. Neoproterozoic fossils in Mesoproterozoic rocks? Chemostratigraphic resolution of a biostratigraphic conundrum from the North China Platform. Precambrian Research, 84: 197-220. 
Young, G.M. 1979. Correlation of middle and upper Proterozoic strata of the northern rim of the North Atlantic craton. Transactions of the Royal Society of Edinburgh, 70: 323-336.

Young, G.M. 1981. Upper Proterozoic supracrustal rocks of North America: a brief review. Precambrian Research, 15: 305-330.
Zartman, R.E., Peterman, Z.E., Obradovich, J.D., Gallego, M.D., and Bishop, D.T. 1982. Age of the Cressport C sill near Estport, Idaho. In Society of Economic Geologists' Coeur d'Alene Field Conference. Edited by R.R. Reid and G.A. Williams. Idaho Bureau of Mines and Geology, Bulletin 24, pp. 61-69. 